\title{
Interval Type-2 Fuzzy Logic Systems Made Simple
}

\author{
Jerry M. Mendel, Life Fellow, IEEE, Robert I. John, Member, IEEE, and Feilong Liu, Student Member, IEEE
}

\begin{abstract}
To date, because of the computational complexity of using a general type-2 fuzzy set (T2 FS) in a T2 fuzzy logic system (FLS), most people only use an interval T2 FS, the result being an interval T2 FLS (IT2 FLS). Unfortunately, there is a heavy educational burden even to using an IT2 FLS. This burden has to do with first having to learn general T2 FS mathematics, and then specializing it to an IT2 FSs. In retrospect, we believe that requiring a person to use T2 FS mathematics represents a barrier to the use of an IT2 FLS. In this paper, we demonstrate that it is unnecessary to take the route from general T2 FS to IT2 FS, and that all of the results that are needed to implement an IT2 FLS can be obtained using T1 FS mathematics. As such, this paper is a novel tutorial that makes an IT2 FLS much more accessible to all readers of this journal. We can now develop an IT2 FLS in a much more straightforward way.
\end{abstract}

Index Terms-Fuzzy logic system, interval type-2 fuzzy sets, type-2 fuzzy logic system, type-2 fuzzy sets.

\section{INTRODUCTION}

$\mathbf{T}$ YPE-2 fuzzy sets (T2 FSs), originally introduced by Zadeh [24], provide additional design degrees of freedom in Mamdani and TSK fuzzy logic systems (FLSs), which can be very useful when such systems are used in situations where lots of uncertainties are present [18]. The resulting type-2 fuzzy logic systems (T2 FLS) have the potential to provide better performance than a type-1 (T1) FLS (e.g., [4], [10]-[15], [21]-[23]). To-date, because of the computational complexity of using a general T2 FS, most people only use interval T2 FSs in a T2 FLS, the result being an interval T2 FLS (IT2 FLS) [9]. The computations associated with interval T2 FSs are very manageable, which makes an IT2 FLS quite practical [16].

Unfortunately, there is a heavy educational burden even to using an IT2 FLS, namely, one must first become proficient about a T1 FLS (this does not change as a result of this paper), then one must become proficient about general T2 FSs, operations performed upon them (T2 FS mathematics-join, meet, negation), T2 fuzzy relations (extended sup-star composition), and T2 FLSs, after which one can then focus on interval T2 FSs, their associated operations and relations, and IT2 FLSs, all as examples of the more general results. To obtain such a level of proficiency, one has to make a very significant investment of time, something that many practicing engineers do not have.

Manuscript received November 23, 2004; revised June 14, 2005 and October 6, 2005.

J. M. Mendel and F. Liu are with the Signal and Image Processing Institute, the Department of Electrical Engineering, University of Southern California, Los Angeles, CA 90089-2564 USA (e-mail: mendel@ sipi.usc.edu; feilongl@usc.edu).

R. I. John is with the Centre for Computational Intelligence, the Department of Computer Science, Faculty of Computing Science and Engineering, De Montfort University, Leicester LE1 9BH, U.K. (e-mail: rij@dmu.ac.ak)

Digital Object Identifier 10.1109/TFUZZ.2006.879986
In retrospect, we believe that requiring a person to use T2 FS mathematics represents a barrier to the use of IT2 FSs and FLSs. Here, we demonstrate that it is unnecessary to take the above route, from general T2 FS to IT2 FS, and that all of the results that are needed to implement an IT2 FLS can be obtained using T1 FS mathematics. As such, we hope that this paper makes IT2 FLSs much more accessible to all readers of this journal.

In order to make this paper as self-contained as possible, we begin, in Section II, by reviewing the IT2 FS and introducing the terminology of such sets. Set theoretic operations are the building blocks of IT2 FLSs, so in Section III, we provide new derivations of the set theoretic operations of the union of IT2 FSs and the complement of an IT2 FS that are totally within the framework of T1 FS mathematics. Because the derivation of the intersection of IT2 FSs is so similar to that of the union, we do not include it here. We include these derivations here because, as we just mentioned, these set theoretic operations are widely used in an IT2 FLS. In Section IV, we briefly review the basics of a T1 FLS, because the formulas for such a FLS are used in our derivations of the formulas for an IT2 FLS. In Section V we derive all of the formulas that are associated with an IT2 FLS, beginning with the simplest situation, a single rule with one antecedent and a crisp input (singleton fuzzification), so that the reader does not get lost in the notation of the more complicated situations and can focus on how T1 FS mathematics are used to obtain the key results. Then we explain how these simple results can be modified to the more complicated situations when a rule has multiple antecedents but the input is either crisp, or is a T1 FS or a T2 FS, and also how to handle multiple rules. In Section V we also review the centroid of an IT2 FLS, because its calculation is the basis for type-reduction methods that are used in going from fired-rule IT2 FSs to the defuzzified number at the final output of such a FLS. Finally, in Section VI we draw conclusions.

\section{INTERVAL TyPE-2 FuZZy SETS}

In this section (which is similar to Section II in [20]), we define an IT2 FS and some important associated concepts, so as to provide a simple collection of mathematically well-defined terms that will let us effectively communicate about such sets. Our motivation is that this material is used extensively in the rest of the paper. To begin we locate an IT2 FS in the taxonomy of a general T2 FS.

Imagine blurring the type-1 membership function (MF) depicted in Fig. 1(a) by shifting the points on the triangle either to the left or to the right and not necessarily by the same amounts, as in Fig. 1(b). Then, at a specific value of $x$, say $x^{\prime}$, there no longer is a single value for the MF; instead, the MF takes on values wherever the vertical line intersects the blur. Those values need not all be weighted the same; hence, we can assign 


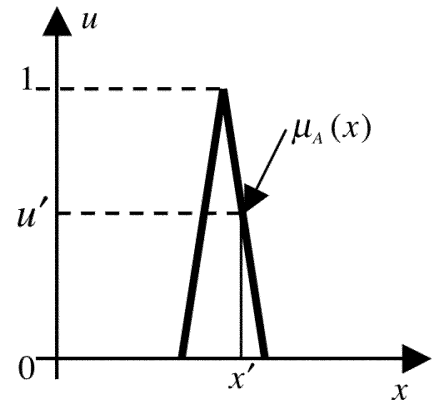

(a)

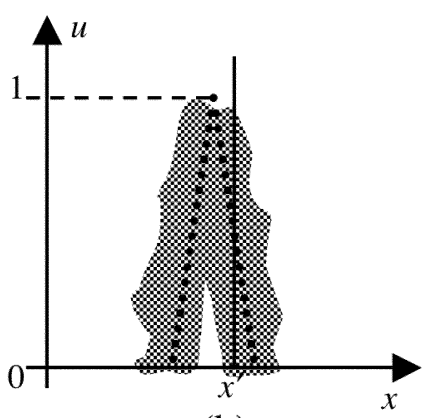

(b)
Fig. 1. (a) Type-1 MF. (b) Blurred type-1 MF.

an amplitude distribution to all of those points. Doing this for all $x \in X$, we create a three-dimensional MF-a T2 MF-that characterizes a T2 FS.

Definition 1: A $T 2 F S$, denoted $\tilde{A}$, is characterized by a type- 2 $M F \mu_{\tilde{A}}(x, u)$, where $x \in X$ and $u \in J_{x} \subseteq[0,1]$, i.e.,

$$
\tilde{A}=\left\{\left((x, u), \mu_{\tilde{A}}(x, u)\right) \mid \forall x \in X, \forall u \in J_{x} \subseteq[0,1]\right\}
$$

in which $0 \leq \mu_{\tilde{A}}(x, u) \leq 1 . \tilde{A}$ can also be expressed as

$$
\tilde{A}=\int_{x \in X} \int_{u \in J_{x}} \mu_{\tilde{A}}(x, u) /(x, u) \quad J_{x} \subseteq[0,1]
$$

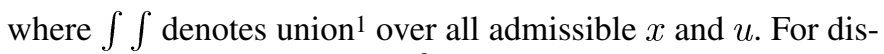
crete universes of discourse, $\int$ is replaced by $\sum$.

In Definition 1, the first restriction that $\forall u \in J_{x} \subseteq[0,1]$ is consistent with the $\mathrm{T} 1$ constraint that $0 \leq \mu_{A}(x) \leq 1$, i.e., when uncertainties disappear a $\mathrm{T} 2 \mathrm{MF}$ must reduce to a $\mathrm{T} 1 \mathrm{MF}$, in which case the variable $u$ equals ${ }^{2} \mu_{A}(x)$ and $0 \leq \mu_{A}(x) \leq 1$. The second restriction that $0 \leq \mu_{\tilde{A}}(x, u) \leq 1$ is consistent with the fact that the amplitudes of a MF should lie between or be equal to 0 and 1 .

Definition 2: When all $\mu_{\tilde{A}}(x, u)=1$ then $\tilde{A}$ is an interval T2 FS (IT2 FS).

Although the third dimension of the general T2 FS is no longer needed ${ }^{3}$ because it conveys no new information about the IT2 FS, the IT2 FS can still be expressed as a special case of the general T2 FS in (2), as

$$
\tilde{A}=\int_{x \in X} \int_{u \in J_{x}} 1 /(x, u), \quad J_{x} \subseteq[0,1] .
$$

In the rest of this paper we will only be interested in IT2 FSs. Note, however, that in order to introduce the remaining widely used terminology of a T2 FS we temporarily continue to retain the third dimension for an IT2 FS.

\footnotetext{
${ }^{1}$ Recall that the union of two sets $A$ and $B$ is by definition another set that contains the elements in either $A$ or $B$. When we view each element of a T2 FS as a subset, then the unions in (2) conform to the classical definition of union, since each element of that set is distinct. At a specific value of $x$ and $u$ only one term is activated in the union.

${ }^{2}$ In this case, the third dimension disappears.

${ }^{3}$ There is equivalence between an IT2 FS and interval-valued fuzzy sets (e.g., [2] and [3]).
}

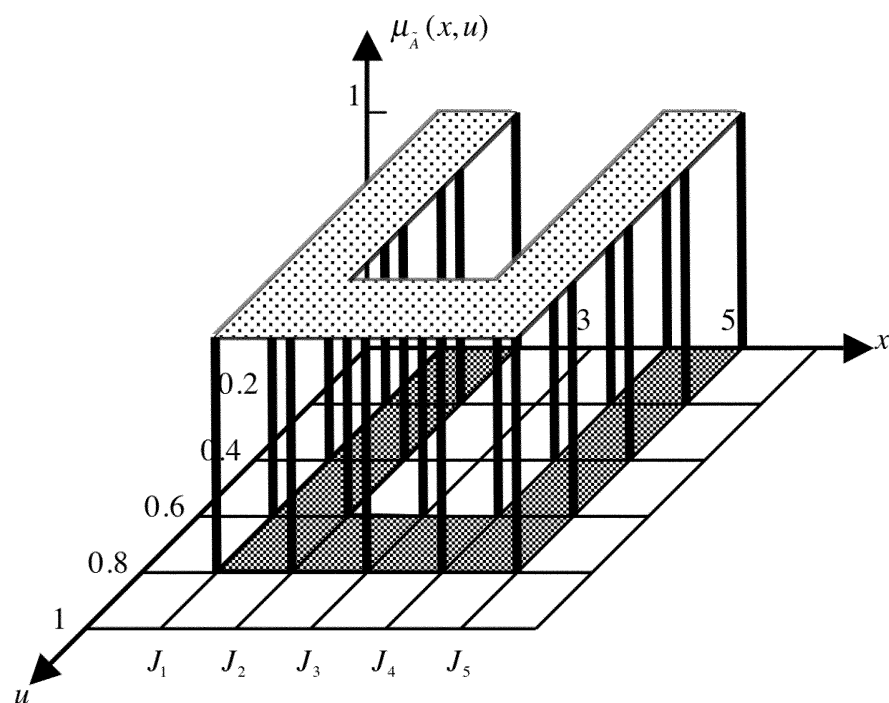

Fig. 2. Example of an interval T2 MF for discrete universes of discourse. The shaded area in the $x-u$ plane is the FOU.

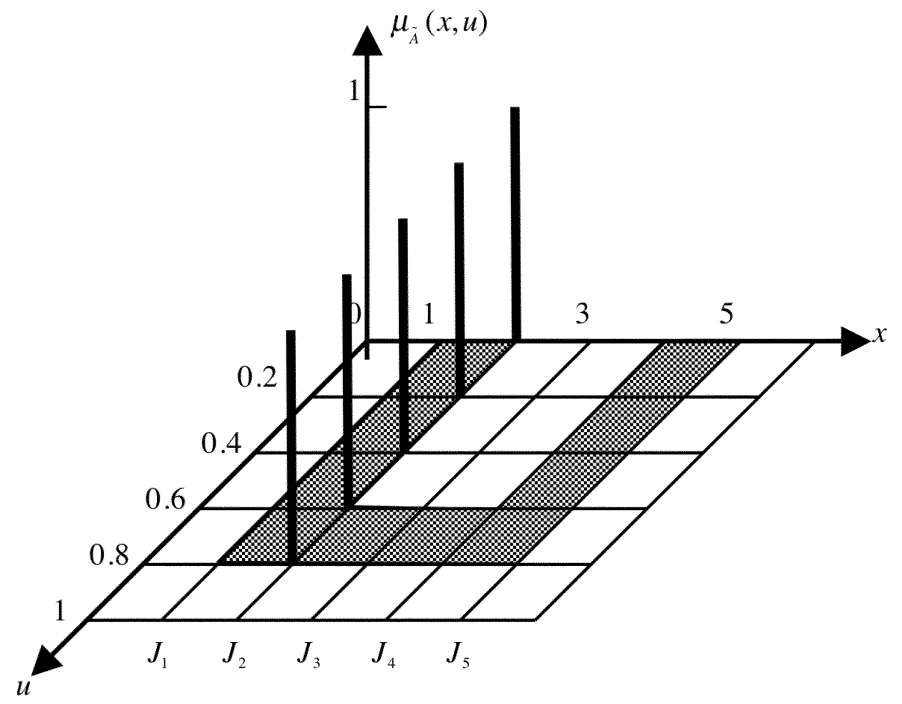

Fig. 3. Example of a vertical slice for the T2 MF depicted in Fig. 2.

Definition 3: At each value of $x$, say $x=x^{\prime}$, the 2-D plane whose axes are $u$ and $\mu_{\tilde{A}}\left(x^{\prime}, u\right)$ is called a vertical slice of $\mu_{\tilde{A}}(x, u)$. A secondary $M F$ is a vertical slice of $\mu_{\tilde{A}}(x, u)$. It is $\mu_{\tilde{A}}\left(x=x^{\prime}, u\right)$ for $x^{\prime} \in X$ and $\forall u \in J_{x^{\prime}} \subseteq[0,1]$, i.e.,

$$
\mu_{\tilde{A}}\left(x=x^{\prime}, u\right) \equiv \mu_{\tilde{A}}\left(x^{\prime}\right)=\int_{u \in J_{x^{\prime}}} 1 / u \quad J_{x^{\prime}} \subseteq[0,1] .
$$

Because $\forall x^{\prime} \in X$, we drop the prime notation on $\mu_{\tilde{A}}\left(x^{\prime}\right)$, and refer to $\mu_{\tilde{A}}(x)$ as a secondary MF; it is a T1 FS, an interval FS, which we also refer to as a secondary set.

Example 1: The T2 MF that is depicted in Fig. 2 has five vertical slices associated with it. The one at $x=2$ is depicted in Fig. 3. The secondary MF at $x=2$ is $\mu_{\tilde{A}}(2)=1 / 0+1 / 0.2+$ $1 / 0.4+1 / 0.6+1 / 0.8$.

Based on the concept of secondary sets, we can reinterpret an IT2 FS as the union (see footnote 1) of all secondary sets, i.e., using (4), we can re-express $\tilde{A}$ in a vertical-slice manner, as

$$
\tilde{A}=\left\{\left(x, \mu_{\tilde{A}}(x)\right) \mid \forall x \in X\right\}
$$


or, alternatively, as

$\tilde{A}=\int_{x \in X} \mu_{\tilde{A}}(x) / x=\int_{x \in X}\left[\int_{u \in J_{x}} 1 / u\right] / x, \quad J_{x} \subseteq[0,1]$

Definition 4: The domain of a secondary MF is called the primary membership of $x$. In (6), $J_{x}$ is the primary membership of $x$, where $J_{x} \subseteq[0,1]$ for $\forall x \in X$.

Definition 5: The amplitude of a secondary MF is called a secondary grade. The secondary grades of an IT2 FS are all equal to 1 .

If $X$ and $J_{x}$ are both discrete (either by problem formulation-as in Example 1-or by discretization of continuous universes of discourse), then the right-most part of (6) can be expressed as

$$
\begin{aligned}
\tilde{A} & =\sum_{x \in X}\left[\sum_{u \in J_{x}} 1 / u\right] / x \\
& =\sum_{i=1}^{N}\left[\sum_{u \in J_{x_{i}}} 1 / u\right] / x_{i} \\
& =\left[\sum_{k=1}^{M_{1}} 1 / u_{1 k}\right] / x_{1}+\cdots+\left[\sum_{k=1}^{M_{N}} 1 / u_{N k}\right] / x_{N} .
\end{aligned}
$$

In this equation, + also denotes union. Observe that $x$ has been discretized into $N$ values and at each of these values $u$ has been discretized into $M_{i}$ values. The discretization along each $u_{i k}$ does not have to be the same, which is why we have shown a different upper sum for each of the bracketed terms; however, if the discretization of each $u_{i k}$ is the same, then $M_{1}=M_{2}=$ $\cdots=M_{N} \equiv M$.

Example 1 (Continued): In Fig. 2, the union of the five secondary MFs at $x=1,2,3,4,5$ is $\mu_{\tilde{A}}(x, u)$. Observe that the primary memberships are

$$
\begin{aligned}
& J_{1}=J_{2}=J_{4}=J_{5}=\{0,0.2,0.4,0.6,0.8\} \text { and } \\
& J_{3}=\{0.6,0.8\}
\end{aligned}
$$

and we have only included values in $J_{3}$ for which $\mu_{\tilde{A}}(x, u) \neq 0$. Each of the spikes in Fig. 2 represents $\mu_{\tilde{A}}(x, u)$ at a specific $(x, u)$-pair, and its amplitude of 1 is the secondary grade.

Definition 6: Uncertainty in the primary memberships of an IT2 FS, $\tilde{A}$, consists of a bounded region that we call the footprint of uncertainty (FOU). It is the union of all primary memberships, i.e.,

$$
\operatorname{FOU}(\tilde{A})=\bigcup_{x \in X} J_{x}
$$

This is a vertical-slice representation of the FOU, because each of the primary memberships is a vertical slice.

The shaded region on the $x-u$ plane in Fig. 2 is the FOU. Because the secondary grades of an IT2 FS convey no new information, the FOU is a complete description of an IT2 FS. The uniformly shaded FOU of an IT2 FS denotes that there is a uniform distribution that sits on top of it. The uniformly blurred T1 FS in Fig. 1(b) is another example of the FOU of an IT2 FS.
Comment: Mendel and John [20] introduced the concept of the domain of uncertainty (DOU) for a T2 FS, $\tilde{A}$, as the union of all the primary memberships of $\tilde{A}$, i.e., $\operatorname{DOU}(\tilde{A})=\bigcup_{x \in X} J_{x}$. They did so in order to distinguish between primary variables that are or are not naturally ordered, ${ }^{4}$ and T2 FSs that are either discrete, continuous, or hybrid. 5 If a T2 FS is continuous with a naturally ordered primary variable, as in this paper, then $\operatorname{DOU}(\tilde{A})=\operatorname{FOU}(\tilde{A})$. If a T2 FS is discrete with a naturally ordered primary variable, also as in this paper (e.g., Fig. 2), then it is technically more correct to use $\operatorname{DOU}(\tilde{A})$ because a shaded region (which, in this case, is an artistic liberty) implies the existence of all points in it, but for discrete universes of discourse only a finite number of separate points exist in it; however, because the term FOU is already so well entrenched in the T2 literature, we will continue to use $\operatorname{FOU}(\tilde{A})$ for both cases.

Definition 7: The upper membership function (UMF) and lower membership function (LMF) of $\tilde{A}$ are two T1 MFs that bound the FOU (e.g., see Fig. 4). The UMF is associated with the upper bound of $\operatorname{FOU}(\tilde{A})$ and is denoted $\bar{\mu}_{\tilde{A}}(x), \forall x \in X$, and the LMF is associated with the lower bound of $\operatorname{FOU}(\tilde{A})$ and is denoted $\underline{\mu}_{\tilde{A}}(x), \forall x \in X$, i.e.,

$$
\begin{aligned}
\bar{\mu}_{\tilde{A}}(x) \equiv \overline{\operatorname{FOU}(\tilde{A})} & \forall x \in X \\
\underline{\mu}_{\tilde{A}}(x) \equiv \underline{\operatorname{FOU}(\tilde{A})} & \forall x \in X .
\end{aligned}
$$

Note that for an IT2 FS $J_{x}=\left[\underline{\mu}_{\tilde{A}}(x), \bar{\mu}_{\tilde{A}}(x)\right], \forall x \in X$.

Definition 8: For discrete universes of discourse $X$ and $U$, an embedded IT2 FS has $N$ elements, where $\tilde{A}_{e}$ contains exactly one element from $J_{x_{1}}, J_{x_{2}}, \ldots$, and $J_{x_{N}}$, namely $u_{1}, u_{2}, \ldots$, and $u_{N}$, each with a secondary grade equal to 1 , i.e.,

$$
\tilde{A}_{e}=\sum_{i=1}^{N}\left[1 / u_{i}\right] / x_{i} \quad u_{i} \in J_{x_{i}} \subseteq U=[0,1] .
$$

Set $\tilde{A}_{e}$ is embedded in $\tilde{A}$, and, there are a total ${ }^{6}$ of $\prod_{i=1}^{N} M_{i} \tilde{A}_{e}$.

An example of an embedded IT2 FS is depicted in Fig. 4; it is the wavy curve for which its secondary grades (not shown) are all equal to 1 . Other examples of $\tilde{A}_{e}$ are $1 / \bar{\mu}_{\tilde{A}}(x)$ and $1 / \underline{\mu}_{\tilde{A}}(x)$, $\forall x \in X$, where it is understood that in this notation the secondary grade equals 1 at all values of $\bar{\mu}_{\tilde{A}}(x)$ and $\underline{\mu}_{\tilde{A}}(x)$.

Definition 9: For discrete universes of discourse $X$ and $U$, an embedded T1 FS $A_{e}$ has $N$ elements, one each from $J_{x_{1}}, J_{x_{2}}, \ldots$, and $J_{x_{N}}$, namely $u_{1}, u_{2}, \ldots$, and $u_{N}$, i.e.,

$$
A_{e}=\sum_{i=1}^{N} u_{i} / x_{i} \quad u_{i} \in J_{x_{i}} \subseteq U=[0,1]
$$

${ }^{4}$ Examples of primary variables that are (are not) naturally ordered are temperature, pressure, height, etc. (beautiful, ill, happy, etc.).

${ }^{5} \mathrm{~A}$ T2 FS is discrete if the primary variable $x$ takes discrete values and the secondary MFs are also discrete. It is continuous if the primary variable $x$ is from a continuous domain and all the secondary MFs are also continuous. It is hybrid if the values of the primary variable $x$ are discrete (continuous) and the secondary MFs are continuous (discrete).

${ }^{6}$ For a continuous IT2 FS, although there are an uncountable number of embedded IT2 FSs, the concept of an embedded IT2 FS (as well as of an embedded T1 FS (Def. 9)) is still a theoretically useful one. 


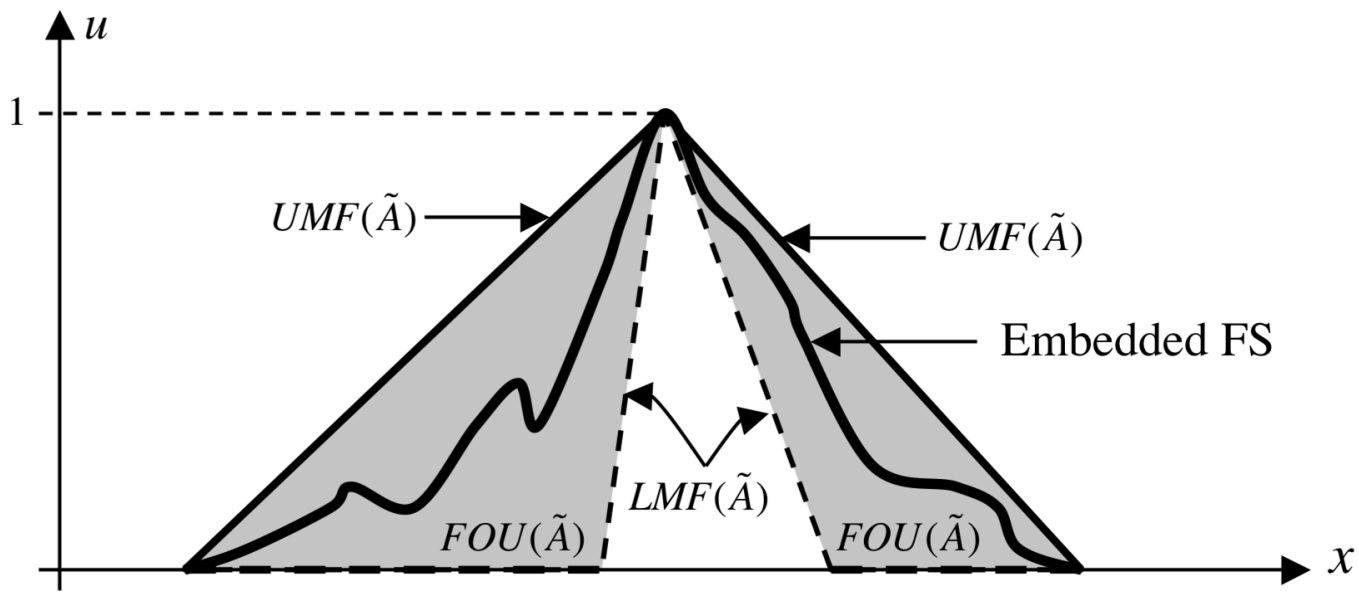

Fig. 4. FOU (shaded), LMF (dashed), UMF (solid) and an embedded FS (wavy line) for IT2 FS $\tilde{A}$.

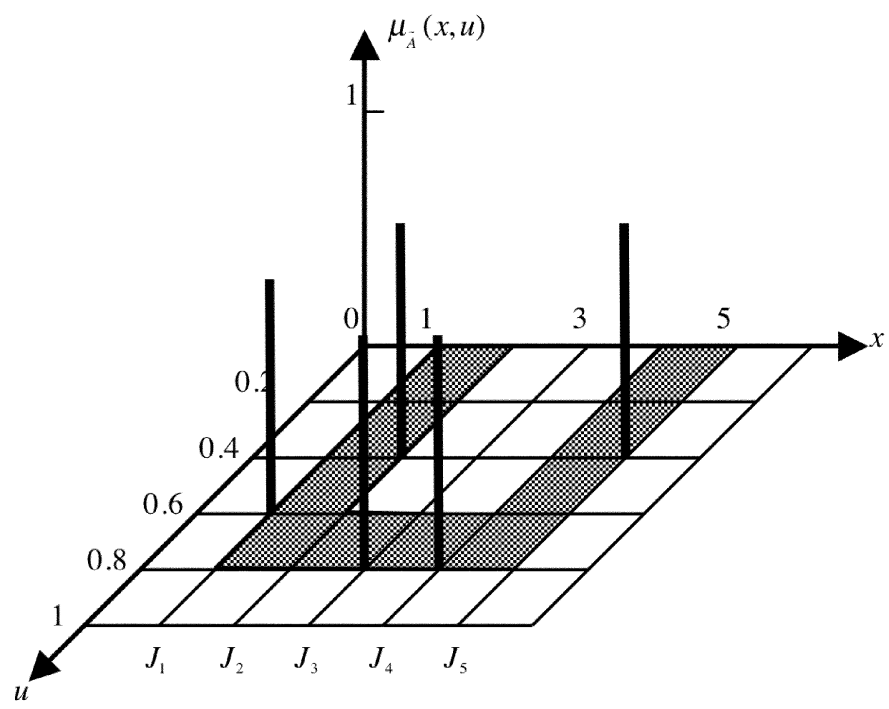

Fig. 5. Example of an embedded IT2 FS associated with the T2 MF depicted in Fig. 2.

Set $A_{e}$ is the union of all the primary memberships of set $\tilde{A}_{e}$ in (11), and, there are a total of $\prod_{i=1}^{N} M_{i} A_{e}$. Note that $A_{e}$ acts as the domain for $\tilde{A}_{e}$.

An example of an embedded T1 FS is depicted in Fig. 4; it is the wavy curve. Other examples of $A_{e}$ are $\bar{\mu}_{\tilde{A}}(x)$ and $\underline{\mu}_{\tilde{A}}(x)$, $\forall x \in X$.

Example 2: Fig. 5 depicts one of the possible $5^{4} \times 2=1,250$ embedded IT2 FSs for the T2 MF that is depicted in Fig. 2. Observe that the embedded T1 FS that is associated with this embedded IT2 FS is $A_{e}=0.6 / 1+0.4 / 2+0.8 / 3+0.8 / 4+$ $0.4 / 5$.

Comparing (11) and (12), we see that the embedded IT2 FS $\tilde{A}_{e}$ can be represented in terms of the embedded T1 FS $A_{e}$, as

$$
\tilde{A}_{e}=1 / A_{e}
$$

with the understanding that this means putting a secondary grade of 1 at all points of $A_{e}$. We will make heavy use of this new way to represent $\tilde{A}_{e}$ in the sequel.
So far we have emphasized the vertical-slice representation (decomposition) of an IT2 FS as given in (6). Next, we provide a different representation for such a fuzzy set that is in terms of so-called wavy slices. This representation, which makes very heavy use of embedded IT2 FSs (Definition 8), was first presented in [19] for an arbitrary T2 FS, and is the bedrock for the rest of this paper. We state this result for a discrete IT2 FS.

Theorem 1 (Representation Theorem): For an IT2 FS, for which $X$ and $U$ are discrete, $\tilde{A}$ is the union of all of its embedded IT2 FSs, i.e.,

$$
\tilde{A}=\sum_{j=1}^{n_{A}} \tilde{A}_{e}^{j}
$$

where $\left(j=1, \ldots, n_{A}\right)$

$$
\tilde{A}_{e}^{j}=\sum_{i=1}^{N}\left[1 / u_{i}^{j}\right] / x_{i} \quad u_{i}^{j} \in J_{x_{i}} \subseteq U=[0,1]
$$

and

$$
n_{A}=\prod_{i=1}^{N} M_{i}
$$

in which $M_{i}$ denotes the discretization levels of secondary variable $u_{i}^{j}$ at each of the $N x_{i}$.

Comment 1: This theorem expresses $\tilde{A}$ as a union of simpler T2 FSs, the $\tilde{A}_{e}^{j}$. They are simpler because their secondary MFs are singletons. Whereas (6) is a vertical slice representation of $\tilde{A},(14)$ is a wavy slice representation of $\tilde{A}$.

Comment 2: A detailed proof of this theorem appears in [19]. Although it is important to have such a proof, we maintain that the results in (14) are obvious using the following simple geometric argument.

- The MF of an IT2 FS is three-dimensional (3-D) (e.g., Fig. 2). Each of its embedded IT2 FSs is a 3-D wavy slice (a foil). Create all of the possible wavy slices and take their union to reconstruct the original 3-D MF. Same points, which occur in different wavy slices, only appear once in the set-theoretic union. 
With reference to Fig. 4, (14) means collecting all of the embedded IT2 FSs into a bundle of such T2 fuzzy sets. Equivalently, because of (13), we can collect all of the embedded T1 FSs into a bundle of such T1 FSs.

Corollary 1: Because all of the secondary grades of an IT2 FS equal 1, we can also express (14) and (15) as

$$
\tilde{A}=1 / \operatorname{FOU}(\tilde{A})
$$

where

$$
\operatorname{FOU}(\tilde{A})=\sum_{j=1}^{n_{A}} A_{e}^{j}= \begin{cases}\left\{\underline{\mu}_{\tilde{A}}(x), \ldots, \bar{\mu}_{\tilde{A}}(x)\right\} & \forall x \in X_{d} \\ {\left[\underline{\mu}_{\tilde{A}}(x), \bar{\mu}_{\tilde{A}}(x)\right]} & \forall x \in X\end{cases}
$$

and [see (12)]

$$
A_{e}^{j}=\sum_{i=1}^{N} u_{i}^{j} / x_{i} \quad u_{i}^{j} \in J_{x_{i}} \subseteq U=[0,1] .
$$

The top line of (18) is for a discrete universe of discourse, $X_{d}$, and contains $n_{A}$ elements (functions), where $n_{A}$ is given by (16), and the bottom line is for a continuous universe of discourse and is an interval set of functions, meaning that it contains an uncountable number of functions that completely fills the space between $\bar{\mu}_{\tilde{A}}(x)-\underline{\mu}_{\tilde{A}}(x)$, for $\forall x \in X$.

Proof: From (13), each $\tilde{A}_{e}^{j}$ in (14) can be expressed as $1 / A_{e}^{j}$; hence

$$
\tilde{A}=\sum_{j=1}^{n_{A}}\left(1 / A_{e}^{j}\right)=1 / \sum_{j=1}^{n_{A}} A_{e}^{j} \equiv 1 / \operatorname{FOU}(\tilde{A})
$$

which is (17). Note that, as already mentioned $\underline{\mu}_{\tilde{A}}(x)$ and $\bar{\mu}_{\tilde{A}}(x)$ are two legitimate elements of the $n_{A}$ elements of $A_{e}^{j}$. In fact, they are the lower and upper bounding functions, respectively, for these $n_{A}$ functions. For discrete universes of discourse, we can therefore express $\operatorname{FOU}(\tilde{A})$ as in the top line of $(18)$, whereas for continuous universes of discourse we can express $\operatorname{FOU}(\tilde{A})$ as in the bottom line of (18).

Equation (18) is a new wavy-slice representation of $\mathrm{FOU}(\tilde{A})$, because all $A_{e}^{j}$ are functions, i.e., they are wavy-slices. We will see in the sequel that we do not need to know the explicit natures of any of the wavy slices in $\operatorname{FOU}(\tilde{A})$ other than $\underline{\mu}_{\tilde{A}}(x)$ and $\bar{\mu}_{\tilde{A}}(x)$.

\section{SET THEORETIC OPERATIONS}

Our goal in this section is to derive formulas for the union and intersection of two IT2 FSs and also the formula for the complement of an IT2 FS, because these operations are widely used in an IT2 FLS. Present approaches to doing this use the Extension Principle [24], alpha-cuts, or interval arithmetic (e.g., [8]). Our approach will be based entirely on Representation Theorem 1, already well-known formulas for the union and intersection of two T1 FSs, and the formula for the complement of a T1 FS.
Theorem 2: a) The union of two IT2 FSs, $\tilde{A}$ and $\tilde{B}$, is

$\tilde{A} \cup \tilde{B}=1 /\left[\underline{\mu}_{\tilde{A}}(x) \vee \underline{\mu}_{\tilde{B}}(x), \bar{\mu}_{\tilde{A}}(x) \vee \bar{\mu}_{\tilde{B}}(x)\right] \quad \forall x \in X$.

b) The intersection of two IT2 FSs, $\tilde{A}$, and $\tilde{B}$, is

$$
\tilde{A} \cap \tilde{B}=1 /\left[\underline{\mu}_{\tilde{A}}(x) \wedge \underline{\mu}_{\tilde{B}}(x), \bar{\mu}_{\tilde{A}}(x) \wedge \bar{\mu}_{\tilde{B}}(x)\right] \quad \forall x \in X .
$$

c) The complement of IT2 FS $\tilde{A}, \overline{\tilde{A}}$, is

$$
\overline{\tilde{A}}=1 /\left[1-\bar{\mu}_{\tilde{A}}(x), 1-\underline{\mu}_{\tilde{A}}(x)\right] \quad \forall x \in X .
$$

Proof: Because the proofs of parts a) and b) are so similar, we only provide the proofs for parts a) and c).

a) Consider two IT2 FSs $\tilde{A}$ and $\tilde{B}$. From Representation Theorem 1 and Corollary 1 , it follows that ${ }^{7}$ :

$$
\begin{aligned}
\tilde{A} \cup \tilde{B} & =\sum_{j=1}^{n_{A}} \tilde{A}_{e}^{j} \cup \sum_{i=1}^{n_{B}} \tilde{B}_{e}^{i}=\sum_{j=1}^{n_{A}} \sum_{i=1}^{n_{B}} \tilde{A}_{e}^{j} \cup \tilde{B}_{e}^{i} \\
& =1 / \operatorname{FOU}(\tilde{A} \cup \tilde{B})
\end{aligned}
$$

where $n_{A}$ and $n_{B}$ denote the number of embedded IT2 FSs that are associated with $\tilde{A}$ and $\tilde{B}$, respectively, and [see the first part of (18)]

$$
\operatorname{FOU}(\tilde{A} \cup \tilde{B})=\sum_{j=1}^{n_{A}} \sum_{i=1}^{n_{B}} A_{e}^{j} \cup B_{e}^{i} .
$$

What we must now do is compute the union of the $n_{A} \times n_{B}$ pairs of embedded T1 FSs $A_{e}^{j}$ and $B_{e}^{i}$. Recall that the union ${ }^{8}$ of two T1 FSs is a function, e.g.,

$$
A_{e}^{j} \cup B_{e}^{i}=\max \left\{\mu_{A_{e}^{j}}\left(x_{k}\right), \mu_{B_{e}^{i}}\left(x_{k}\right)\right\}, \quad k=1,2, \ldots, N .
$$

Consequently, (25) is a collection of $n_{A} \times n_{B}$ functions that contain a lower-bounding function and an upper-bounding function since both $\mu_{A_{e}^{j}}\left(x_{k}\right)$ and $\mu_{B_{e}^{i}}\left(x_{k}\right)$ are bounded for all values of $x_{k}$.

In the case of IT2 FSs, for which each primary membership is defined over a continuous domain, $n_{A}=\infty$ and $n_{B}=\infty$; however, (26) is still true, and the doubly infinite union of embedded T1 FSs in (25) still contains a lower-bounding function and an upper-bounding function, because $\tilde{A}$ and $\tilde{B}$ each have a bounded FOU. We now obtain formulas for these bounding functions.

Recall (see the examples given after Definition 9) that the upper and lower (discrete, or if continuous, sampled) MFs for an IT2 FS are also embedded T1 FSs. For $\tilde{A}, \bar{\mu}_{\tilde{A}}(x)$ and $\underline{\mu}_{\tilde{A}}(x)$ denote its upper MF and lower MF, whereas for $\tilde{B}, \bar{\mu}_{\tilde{B}}(\bar{x})$ and

\footnotetext{
${ }^{7}$ This equation involves summation and union signs. As in the T1 case, where this mixed notation is used, the summation sign is simply shorthand for lots of + signs. The + indicates the union between members of a set, whereas the union sign represents the union of the sets themselves. Hence, by using both the summation and union signs, we are able to distinguish between the union of sets versus the union of members within a set.

${ }^{8}$ Although we present our derivation for maximum, it is also applicable for a general t-conorm.
} 


\section{FLS}

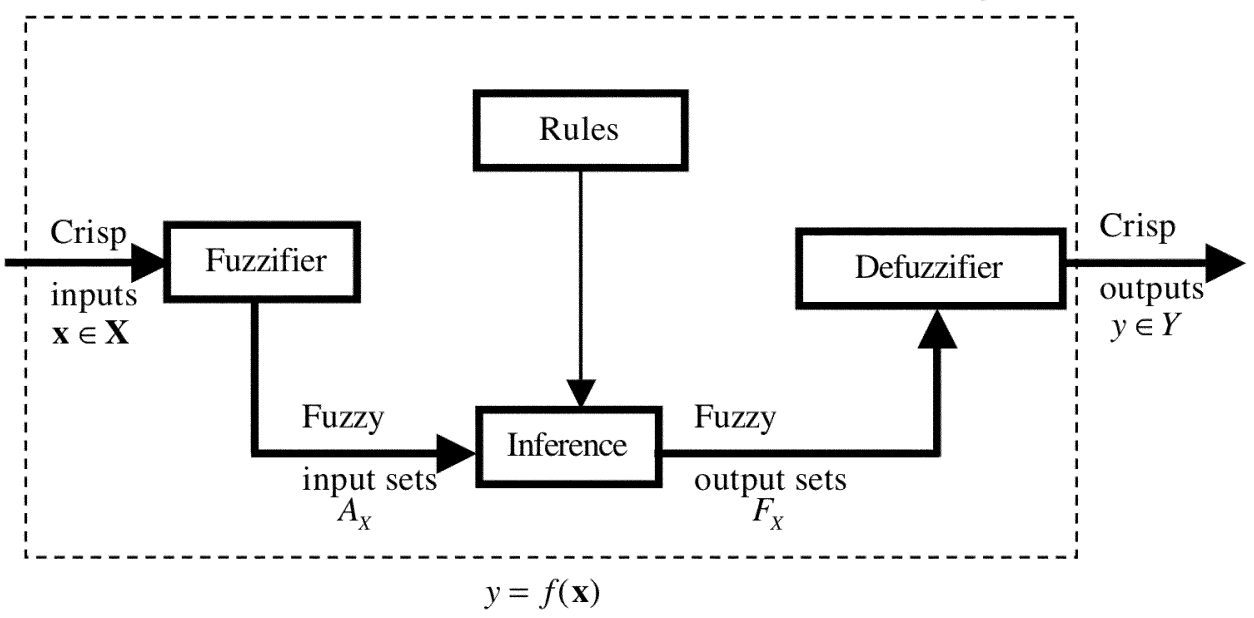

Fig. 6. Type-1 FLS.

$\underline{\mu}_{\tilde{B}}(x)$ denote its comparable quantities. It must, therefore, be true that

$$
\begin{aligned}
& \sup _{\forall j, i} \max \left\{\mu_{A_{e}^{j}}\left(x_{k}\right), \mu_{B_{e}^{i}}\left(x_{k}\right)\right\} \\
& \quad=\max \left\{\bar{\mu}_{\tilde{A}}(x), \bar{\mu}_{\tilde{B}}(x)\right\} \quad \forall x \in X \\
& \quad=\bar{\mu}_{\tilde{A}}(x) \vee \bar{\mu}_{\tilde{B}}(x) \quad \forall x \in X \\
& \inf _{\forall j, i} \max \left\{\mu_{A_{e}^{j}}\left(x_{k}\right), \mu_{B_{e}^{i}}\left(x_{k}\right)\right\} \\
& \quad=\max \left\{\underline{\mu}_{\tilde{A}}(x), \underline{\mu}_{\tilde{B}}(x)\right\} \quad \forall x \in X \\
& \quad=\underline{\mu}_{\tilde{A}}(x) \vee \underline{\mu}_{\tilde{B}}(x) \quad \forall x \in X .
\end{aligned}
$$

From (24)-(28), we conclude that

$$
\begin{aligned}
\tilde{A} \cup \tilde{B} & =1 / \sum_{j=1}^{n_{A}} \sum_{i=1}^{n_{B}} A_{e}^{j} \cup B_{e}^{i} \\
& =1 /\left[\underline{\mu}_{\tilde{A}}(x) \vee \underline{\mu}_{\tilde{B}}(x), \bar{\mu}_{\tilde{A}}(x) \vee \bar{\mu}_{\tilde{B}}(x)\right]
\end{aligned}
$$

which agrees with results that appear in the T2 FS literature (e.g., [17]); however, we have derived (29) entirely within the framework of Representation Theorem 1, Corollary 1 and wavy slices, and did not have to use any T2 FS mathematics to obtain it.

c) Starting with (14), and Corollary 1, we see that

$$
\overline{\tilde{A}}=\overline{\sum_{j=1}^{n_{A}} \tilde{A}_{e}^{j}}=\sum_{j=1}^{n_{A}} \overline{\tilde{A}}_{e}^{j}=1 / \operatorname{FOU}(\overline{\tilde{A}})
$$

where [focusing on continuous universes of discourse; see also the second line of (18)]

$$
\operatorname{FOU}(\overline{\tilde{A}})=\sum_{j=1}^{n_{A}} \bar{A}_{e}^{j}=\left[\underline{\mu}_{\overline{\tilde{A}}}(x), \bar{\mu}_{\overline{\tilde{A}}}(x)\right] \quad \forall x \in X .
$$

Using the well-known fact that the MF of the complement of T1 FS $A$ is $1-\mu_{A}(x)$, it follows that

$$
\mu_{\bar{A}_{e}^{j}}(x)=1-\mu_{A_{e}^{j}}(x) .
$$

Equation (31) is a bundle of functions that has a lower bounding $\left[\underline{\mu}_{\bar{A}}(x)\right]$ and an upper bounding $\left[\bar{\mu}_{\overline{\tilde{A}}}(x)\right]$ function; hence

$$
\begin{aligned}
\bar{\mu}_{\tilde{\tilde{A}}}(x) & =\sup _{\forall j}\left[1-\mu_{A_{e}^{j}}\left(x_{k}\right)\right] \\
& =1-\underline{\mu}_{\tilde{A}}(x) \quad \forall x \in X \\
\underline{\mu}_{\overline{\tilde{A}}}(x) & =\inf _{\forall j}\left[1-\mu_{A_{e}^{j}}\left(x_{k}\right)\right] \\
& =1-\bar{\mu}_{\tilde{A}}(x) \quad \forall x \in X .
\end{aligned}
$$

In obtaining the right-hand parts of (33) and (34), we have used the facts that it is always true that $\bar{\mu}_{\tilde{A}}(x) \geq \underline{\mu}_{\tilde{A}}(x)$, consequently, it is always true that $1-\underline{\mu}_{\tilde{A}}(x) \geq 1-\bar{\mu}_{\tilde{A}}(x)$.

From (30), (31), (33), and (34), we conclude that

$$
\overline{\tilde{A}}=1 / \sum_{j=1}^{n_{A}} \bar{A}_{e}^{j}=1 /\left[1-\bar{\mu}_{\tilde{A}}(x), 1-\underline{\mu}_{\tilde{A}}(x)\right] \quad \forall x \in X
$$

which is (23), and also agrees with results that appear in the T2 FS literature, and again we have not had to use any T2 FS mathematics to derive it.

The generalizations of parts a) and b) of Theorem 2 to more than two IT2 FSs follows directly from (21) and (22) and the associative property of T2 FSs, e.g., see the equation at the bottom of the page.

\section{REVIEW OF TYPE-1 FLS}

Because our derivations of equations for an IT2 FLS in Section $\mathrm{V}$ use the equations for a T1 FLS, we provide a brief re-

$$
\tilde{A} \cup \tilde{B} \cup \tilde{C}=1 /\left[\underline{\mu}_{\tilde{A}}(x) \vee \underline{\mu}_{\tilde{B}}(x) \vee \underline{\mu}_{\tilde{C}}(x), \bar{\mu}_{\tilde{A}}(x) \vee \bar{\mu}_{\tilde{B}}(x) \vee \bar{\mu}_{\tilde{C}}(x)\right] \quad \forall x \in X
$$




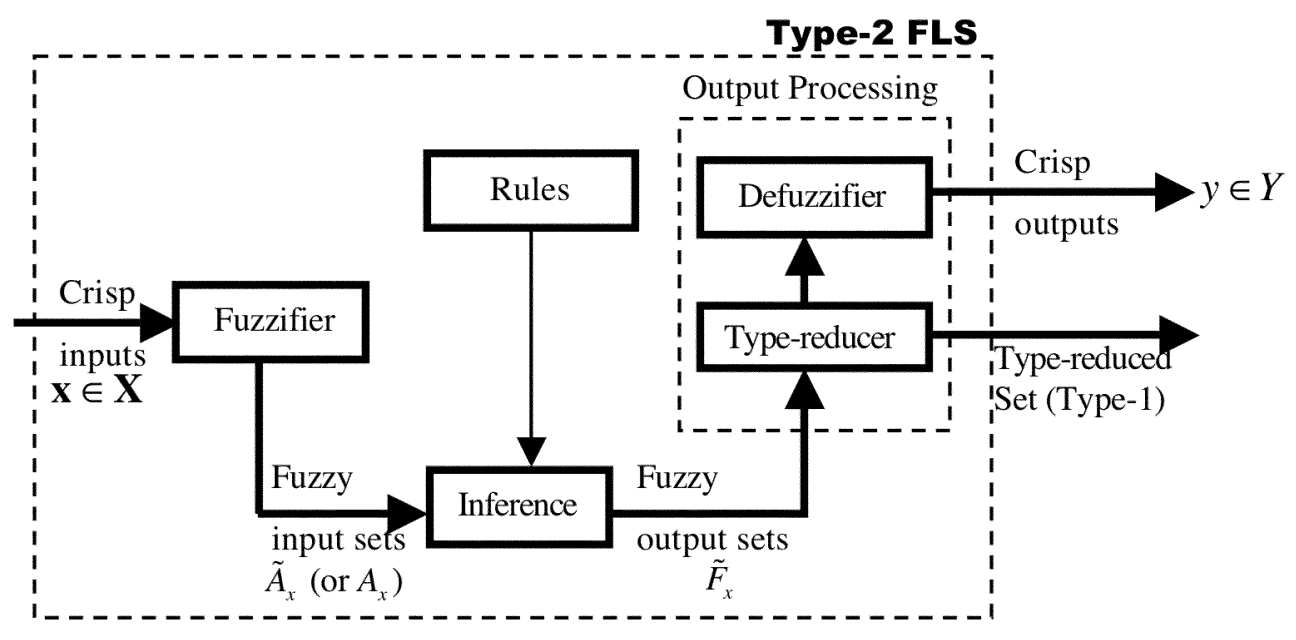

Fig. 8. Type-2 FLS

view of the latter here. A T1 FLS is depicted in Fig. 6. Recall that this FLS is also known as a Mamdani FLS (or fuzzy-rulebased system, fuzzy expert system, fuzzy model, fuzzy system, FL controller [5], [6]). In general, this FLS has $p$ inputs $x_{1} \in$ $X_{1}, \ldots, x_{p} \in X_{p}$, and one output $y \in Y$, and is characterized by $M$ rules, where the $l$ th rule has the form

$$
\begin{array}{r}
R^{l}: \operatorname{IF} x_{1} \text { is } F_{1}^{l} \text { and } \cdots \text { and } x_{p} \text { is } F_{p}^{l}, \\
\qquad \operatorname{THEN} y \text { is } G^{l}, \quad l=1, \ldots, M .
\end{array}
$$

This rule represents a type-1 fuzzy relation between the input space $X_{1} \times \cdots \times X_{p}$ and the output space, $Y$, of the FLS. In the fuzzy inference engine (which is labeled Inference in Fig. 6), fuzzy logic principles are used to combine fuzzy IF-THEN rules from the fuzzy rule base into a mapping from fuzzy input sets in $X_{1} \times \cdots \times X_{p}$ to fuzzy output sets in $Y$. Each rule is interpreted as a fuzzy implication. With reference to (36), let $F_{1}^{l} \times \cdots \times F_{p}^{l}=$ $A^{l}$; then, (36) can be re-expressed as

$$
R^{l}: F_{1}^{l} \times \cdots \times F_{p}^{l} \rightarrow G^{l}=A^{l} \rightarrow G^{l}, \quad l=1, \ldots, M .
$$

Rule $R^{l}$ is described by the MF $\mu_{R^{l}}(\mathbf{x}, y)$, where

$$
\mu_{R^{l}}(\mathbf{x}, y)=\mu_{A^{l} \rightarrow G^{l}}(\mathbf{x}, y)
$$

and $\mathbf{x}=\left(x_{1}, \ldots, x_{p}\right)^{T}$. So, $\mu_{R^{l}}(\mathbf{x}, y)=\mu_{R^{l}}\left(x_{1}, \ldots, x_{p}, y\right)$ and

$$
\begin{aligned}
\mu_{R^{l}}(\mathbf{x}, y) & =\mu_{A^{l} \rightarrow G^{l}}(\mathbf{x}, y)=\mu_{F_{1}^{l} \times \cdots \times F_{p}^{l} \rightarrow G^{l}}(\mathbf{x}, y) \\
& =\mu_{F_{1}^{l} \times \cdots \times F_{p}^{l}}(\mathbf{x}) \star \mu_{G^{l}}(y) \\
& =\mu_{F_{1}^{l}}\left(x_{1}\right) \star \cdots \star \mu_{F_{p}^{l}}\left(x_{p}\right) \star \mu_{G^{l}}(y) \\
& =\left[T_{m=1}^{p} \mu_{F_{m}^{l}}\left(x_{m}\right)\right] \star \mu_{G^{l}}(y)
\end{aligned}
$$

where it has been assumed that Mamdani implications are used, multiple antecedents are connected by and (i.e., by t-norms) and $T$ is short for a t-norm.
The $p$-dimensional input to $R^{l}$ is given by the fuzzy set $A_{\mathbf{x}}$ whose MF is that of a fuzzy Cartesian product, i.e.,

$$
\mu_{A_{\mathbf{x}}}(\mathbf{x})=\mu_{X_{1}}\left(x_{1}\right) \star \cdots \star \mu_{X_{p}}\left(x_{p}\right)=T_{m=1}^{p} \mu_{X_{m}}\left(x_{m}\right) .
$$

Each rule $R^{l}$ determines a fuzzy set $B^{l}=A_{\mathrm{x}} \circ R^{l}$ in $Y$ such that when we use Zadeh's sup-star composition, we obtain $(l=$ $1, \ldots, M)$

$$
\begin{aligned}
\mu_{B^{l}}(y) & =\mu_{A_{\mathbf{x}} \circ R^{l}}(y) \\
& =\sup _{\mathbf{x} \in \mathbf{X}}\left[\mu_{A_{\mathbf{x}}}(\mathbf{x}) \star \mu_{A^{l} \rightarrow G^{l}}(\mathbf{x}, y)\right], \quad y \in Y .
\end{aligned}
$$

This equation is the input-output relationship in Fig. 6 between the fuzzy set that excites a one-rule inference engine and the fuzzy set at the output of that engine.

Substituting (39) and (40) into (41), we see that

$$
\begin{aligned}
\mu_{B^{l}}(y)= & \sup _{\mathbf{x} \in \mathbf{X}}\left[\mu_{A_{\mathbf{x}}}(\mathbf{x}) \star \mu_{A^{l} \rightarrow G^{l}}(\mathbf{x}, y)\right] \\
= & \sup _{\mathbf{x} \in \mathbf{X}}\left[T_{m=1}^{p} \mu_{X_{m}}\left(x_{m}\right) \star\left[T_{m=1}^{p} \mu_{F_{m}^{l}}\left(x_{m}\right)\right] \star \mu_{G^{l}}(y)\right] \\
= & \sup _{\mathbf{x} \in \mathbf{X}}\left\{\left[T_{m=1}^{p} \mu_{X_{m}}\left(x_{m}\right) \star \mu_{F_{m}^{l}}\left(x_{m}\right)\right] \star \mu_{G^{l}}(y)\right\} \\
= & \left\{\left[\sup _{x_{1} \in X_{1}} \mu_{X_{1}}\left(x_{1}\right) \mu_{F_{1}^{l}}\left(x_{1}\right)\right] \star \cdots\right. \\
& \left.\star\left[\sup _{x_{p} \in X_{p}} \mu_{X_{p}}\left(x_{p}\right) \mu_{F_{p}^{l}}\left(x_{p}\right)\right]\right\} \\
& \star \mu_{G^{l}}(y), \quad y \in Y .
\end{aligned}
$$

The inputs to a T1 FLS can be a type-0 (i.e., crisp input) or a type-1 FS, where the former is commonly referred to as a singleton input, with associated singleton fuzzification (SF) and the latter is commonly referred to as a nonsingleton input, with associated nonsingleton fuzzification (NSF). For a singleton input

$$
\mu_{X_{i}}\left(x_{i}\right)= \begin{cases}1 & x_{i}=x_{i}^{\prime} \\ 0 & x_{i} \neq x_{i}^{\prime}\end{cases}
$$

Hence, substituting (43) into (42) for SF, (42) can be expressed for both SF and NSF, as $(l=1, \ldots, M)$; see 


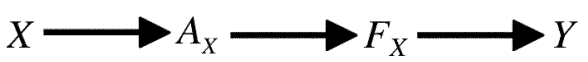

Fig. 7. Mapping from $X$ to $Y$ valid for all rules.

(44) at the bottom of the page. For NSF, we must calculate $\sup _{x_{m} \in X_{m}} \mu_{X_{m}}\left(x_{m}\right) \star \mu_{F_{m}}\left(x_{m}\right)$, i.e., we must first find $x_{m, \max }^{l}$, where

$$
x_{m, \max }^{l}=\arg \sup _{x_{m} \in X_{m}} \mu_{X_{m}}\left(x_{m}\right) \star \mu_{F_{m}^{l}}\left(x_{m}\right)
$$

and then determine $\mu_{X_{m}}\left(x_{m, \text { max }}^{l}\right) \star \mu_{F_{m}^{l}}\left(x_{m, \text { max }}^{l}\right)$. This can be done once MF formulas are specified for $\mu_{X_{m}}\left(x_{m}\right)$ and $\mu_{F_{m}^{l}}\left(x_{m}\right)$ (e.g., [17]).

From a graphical viewpoint, it will be very useful for us in Section V to interpret the flow of the T1 FLS calculations as in Fig. 7.

As is well known, going from the fired rule output FSs in (44) to a number can be accomplished by means of defuzzification (Fig. 6) in many different ways, including9: 1) centroid defuzzification, where first the fired output FSs are unioned and then the centroid of the union is computed; 2) center-of-sums defuzzification, where first the MFs of the fired output FSs are added and then the centroid of the sum is computed; and 3) height, modified height or center-of-sets defuzzification, where properties about the fired rule output FSs (e.g., centroid of consequent FS) are used in a centroid calculation. Regardless of which defuzzification method is chosen, this now completes the chain of calculations for the T1 FLS in Fig. 6.

\section{INTERVAL TYPE-2 FLS}

\section{A. Introduction}

A general T2 FLS is depicted in Fig. 8. It is very similar to the T1 FLS in Fig. 6, the major structural difference being that the defuzzifier block of a T1 FLS is replaced by the output processing block in a T2 FLS. That block consists of type-reduction followed by defuzzification. Type-reduction maps a T2 FS into a T1 FS, and then defuzzification, as usual, maps that T1 FS into a crisp number. Here we assume that all the antecedent and consequent fuzzy sets in rules are T2; however, this need not necessarily be the case in practice. All results remain valid as long as just one FS is T2. This means that a FLS is T2 as long as any one of its antecedent or consequent (or input) FSs is T2.

In the $\mathrm{T} 1$ case, we have rules of the form stated in (36). As just mentioned, the distinction between $\mathrm{T} 1$ and $\mathrm{T} 2$ is associated with

\footnotetext{
${ }^{9}$ Other defuzzification methods such as maximum and mean-of-maxima could also be used; however, in actual applications of a FLS, such defuzzification methods are rarely used.
}

the nature of the MFs, which is not important when forming the rules. The structure of the rules remains exactly the same in the $\mathrm{T} 2$ case, but now some or all of the FSs involved are T2. As for a T1 FLS, the T2 FLS has $p$ inputs $x_{1} \in X_{1}, \ldots, x_{p} \in X_{p}$, and one output $y \in Y$, and, is characterized by $M$ rules, where the $l$ th rule now has the form

$$
\begin{aligned}
& R^{l}: \text { IF } x_{1} \text { is } \tilde{F}_{1}^{l} \text { and } \cdots \text { and } x_{p} \text { is } \tilde{F}_{p}^{l}, \\
& \quad \text { THEN } y \text { is } \tilde{G}^{l}, \quad l=1, \ldots, M .
\end{aligned}
$$

When all of the antecedent and consequent $\mathrm{T} 2$ fuzzy sets are IT2 FSs, then we call the resulting T2 FLS an interval T2 FLS (IT2 FLS). These are the FLSs that we focus on in the rest of this paper.

In order to see the forest from the trees, so-to-speak, we will focus initially on a single rule (i.e., $l=1$ ) that has one antecedent and that is activated by a crisp number (i.e., SF), after which we shall show how those results can be extended first to multiple antecedents, then to NSF, and finally to multiple rules. Because a T2 FLS affords us with the opportunity for either a T1 FS or a T2 FS input (in our case, it will be an IT2 FS), we consider these two different kinds of nonsingleton input situations separately

\section{B. Singleton Fuzzification and One Antecedent}

In the rule 10

$$
\text { IF } x_{1} \text { is } \tilde{F}_{1} \text {, THEN } y \text { is } \tilde{G}
$$

let $\tilde{F}_{1}$ be an IT2 FS in the discrete universe of discourse $X_{1 d}$ for the antecedent, and $\tilde{G}$ be an IT2 FS in the discrete universe of discourse $Y_{d}$ for the consequent. Decompose $\tilde{F}_{1}$ into $n_{F_{1}}$ embedded IT2 FSs $\tilde{F}_{1 e}^{j_{1}}\left(j_{1}=1, \ldots, n_{F_{1}}\right)$, whose domains are the embedded T1 FSs $F_{1 e}^{j_{1}}$, and decompose $(\tilde{G})$ into $n_{G}$ embedded IT2 FSs $\tilde{G}_{e}^{j}\left(j=1, \ldots, n_{G}\right)$, whose domains are the embedded T1 FSs $G_{e}^{j}$. According to (14) of Representation Theorem 1 and Corollary 1 , we see that $\tilde{F}_{1}$ and $\tilde{G}$ can be expressed as:

$$
\tilde{F}_{1}=\sum_{j_{1}=1}^{n_{F_{1}}} \tilde{F}_{1 e}^{j_{1}}=1 / \operatorname{FOU}\left(\tilde{F}_{1}\right)
$$

where

$$
\begin{aligned}
& \operatorname{FOU}\left(\tilde{F}_{1}\right)=\sum_{j_{1}=1}^{n_{F_{1}}} F_{1 e}^{j_{1}} \\
&=\sum_{j_{1}=1}^{n_{F_{1}}} \sum_{i=1}^{N_{x_{1}}} u_{1 i}^{j_{1}} / x_{1 i} \\
& u_{1 i}^{j_{1}} \in J_{x_{1 i}} \subseteq U=[0,1]
\end{aligned}
$$

\footnotetext{
${ }^{10}$ Although it is unnecessary to use the subscript 1 on $x$ for a single-antecedent rule, by doing so we will make the multiple-antecedent case easier to understand because we will understand where the subscript 1 appears in all of the notation and formulas.
}

$$
\mu_{B^{l}}(y)= \begin{cases}{\left[T_{m=1}^{p} \mu_{F_{m}^{l}}\left(x_{m}^{\prime}\right)\right] \star \mu_{G^{l}}(y),} & \mathrm{SF} \\ {\left[T_{m=1}^{p}\left(\sup _{x_{m} \in X_{m}} \mu_{X_{m}}\left(x_{m}\right) \star \mu_{F_{m}^{l}}\left(x_{m}\right)\right)\right] \star \mu_{G^{l}}(y),} & \mathrm{NSF}\end{cases}
$$




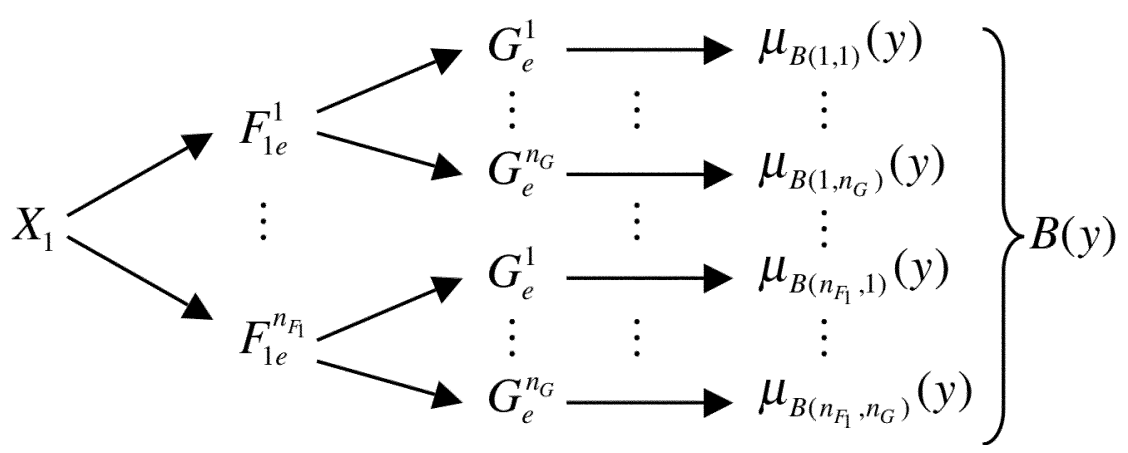

Fig. 9. Fired output FSs for all possible $n_{B}=n_{F_{1}} \times n_{G}$ combinations of the embedded T1 antecedent and consequent FSs for a single antecedent rule.

and

$$
\tilde{G}=\sum_{j=1}^{n_{G}} \tilde{G}_{e}^{j}=1 / \operatorname{FOU}(\tilde{G})
$$

where

$$
\begin{aligned}
\operatorname{FOU}(\tilde{G}) & =\sum_{j=1}^{n_{G}} G_{e}^{j}=\sum_{j=1}^{n_{G}} \sum_{k=1}^{N_{y}} w_{k}^{j} / y_{k}, \\
w_{k}^{j} & \in J_{y_{k}} \subseteq U=[0,1] .
\end{aligned}
$$

Consequently, we have $n_{F_{1}} \times n_{G}$ possible combinations of embedded T1 antecedent and consequent FSs so that the totality of fired output sets for all possible combinations of these embedded T1 antecedent and consequent FSs will be a bundle of functions $B(y)$ as depicted in Fig. 9, where

$$
B(y) \triangleq \sum_{j_{1}=1}^{n_{F_{1}}} \sum_{j=1}^{n_{G}} \mu_{B\left(j_{1}, j\right)}(y) \quad \forall y \in Y_{d}
$$

in which the summations denote union. The relationship between the bundle of functions $B(y)$ in (52) and the FOU of the T2 fired output FS is summarized by the following theorem.

Theorem 3: The bundle of functions $B(y)$ in (52), computed using T1 FS mathematics, is the same as the FOU of the T2 fired output FS, which is computed using T2 FS mathematics. The specific connections are given in (60), (61), (57), and (58).

Proof: From Fig. 9, we see that the fired output of the combination of the $j_{1}$ th embedded T1 antecedent FS and the $j$ th embedded T1 consequent FS can be computed for SF using Mamdani implication as in the top line of (44) with $p=1$, i.e., ${ }^{11}$

$$
\mu_{B\left(j_{1}, j\right)}(y)=\mu_{F_{1 e}^{j_{1}}}\left(x_{1}^{\prime}\right) \star \mu_{G_{e}^{j}}(y) \quad \forall y \in Y_{d} .
$$

Since for any $j_{1}$ and $j, \mu_{B\left(j_{1}, j\right)}(y)$ in (53) is bounded in $[0,1]$, $B(y)$ in (52) must also be a bounded function in $[0,1]$, which means that (52) can be expressed as ${ }^{12}$ :

$$
B(y) \equiv\left\{\underline{\mu}_{\tilde{B}}(y), \ldots, \bar{\mu}_{\tilde{B}}(y)\right\} \quad \forall y \in Y_{d}
$$

\footnotetext{
${ }^{11}$ In (44), the superscript $l$ denotes rule number. Since we are focusing on a single rule, we do not use this superscript here. Our superscripts are associated with specific embedded T1 FSs.

${ }^{12} \mathrm{We}$ choose to call the lower and upper bounding functions in $B(y) \underline{\mu}_{\tilde{B}}(y)$ and $\bar{\mu}_{\tilde{B}}(y)$ rather than $\mu_{B}(y)$ and $\bar{\mu}_{B}(y)$ because doing so will let us more easily connect our T1 FS derivation with the already known IT2 FS results.
}

a set of $n_{F_{1}} \times n_{G}$ functions, where

$$
\begin{array}{ll}
\underline{\mu}_{\tilde{B}}(y)=\inf _{\forall j_{1}, j}\left(\mu_{B\left(j_{1}, j\right)}(y)\right) & \forall y \in Y_{d} \\
\bar{\mu}_{\tilde{B}}(y)=\sup _{\forall j_{1}, j}\left(\mu_{B\left(j_{1}, j\right)}(y)\right) & \forall y \in Y_{d}
\end{array}
$$

denote the lower bounding and upper bounding functions of $B(y)$, respectively.

Let $\bar{\mu}_{\tilde{F}_{1}}\left(x_{1}\right)$ and $\underline{\mu}_{\tilde{F}_{1}}\left(x_{1}\right)$ denote the upper and lower MFs for $\tilde{F}_{1}$, and $\bar{\mu}_{\tilde{G}}(y)$ and $\underline{\mu}_{\tilde{G}}(y)$ denote the upper and lower MFs for $\tilde{G}$. Additionally, let $\bar{\mu}_{F_{1}}\left(x_{1}\right)$ and $\underline{\mu}_{F_{1}}\left(x_{1}\right)$ denote the embedded T1 FSs associated with $\bar{\mu}_{\tilde{F}_{1}}\left(x_{1}\right)$ and $\underline{\mu}_{\tilde{F}_{1}}\left(x_{1}\right)$, respectively, and $\bar{\mu}_{G}(y)$ and $\underline{\mu}_{G}(y)$ denote the corresponding embedded T1 FSs of $\bar{\mu}_{\tilde{G}}(y)$ and $\underline{\mu}_{\tilde{G}}(y)$, respectively. From (53), we see that to compute the infimum of $\mu_{B\left(j_{1}, j\right)}(y)$ we need to choose the smallest embedded T1 FS of both the antecedent and consequent, namely $\underline{\mu}_{F_{1}}\left(x_{1}\right)$ and $\underline{\mu}_{G}(y)$, respectively. By doing this, we obtain the following equation for $\underline{\mu}_{\tilde{B}}(y)$ :

$\underline{\mu}_{\tilde{B}}(y)=\inf _{\forall j_{1}, j}\left(\mu_{B\left(j_{1}, j\right)}(y)\right)=\underline{\mu}_{F_{1}}\left(x_{1}^{\prime}\right) \star \underline{\mu}_{G}(y) \quad \forall y \in Y_{d}$.

Similarly, to compute the supremum of $\mu_{B\left(j_{1}, j\right)}(y)$, we need to choose the largest embedded T1 FS of both the antecedent and consequent, namely $\bar{\mu}_{F_{1}}\left(x_{1}\right)$ and $\bar{\mu}_{G}(y)$, respectively. By doing this, we obtain the following equation for $\bar{\mu}_{\tilde{B}}(y)$ :

$$
\bar{\mu}_{\tilde{B}}(y)=\sup _{\forall j_{1}, j}\left(\mu_{B\left(j_{1}, j\right)}(y)\right)=\bar{\mu}_{F_{1}}\left(x_{1}^{\prime}\right) \star \bar{\mu}_{G}(y) \quad \forall y \in Y_{d} .
$$

Obviously, when the sample rate becomes infinite, the sampled universes of discourse $X_{1 d}$ and $Y_{d}$ can be considered as the continuous universes of discourse $X_{1}$ and $Y$, respectively. In this case, $B(y)$ contains an infinite and uncountable number of elements, which will still be bounded below and above by $\underline{\mu}_{\tilde{B}}(y)$ and $\bar{\mu}_{\tilde{B}}(y)$, respectively, where these functions are still given by (57) and (58) (with $Y_{d} \rightarrow Y$ ), such that (54) can be expressed as

$$
B(y)=\left[\underline{\mu}_{\tilde{B}}(y), \bar{\mu}_{\tilde{B}}(y)\right] \quad \forall y \in Y .
$$

Comparing (59) and the second line of (18), we see that

$$
B(y)=\operatorname{FOU}(\tilde{B})
$$


where

$$
\operatorname{FOU}(\tilde{B})=\left[\underline{\mu}_{\tilde{B}}(y), \bar{\mu}_{\tilde{B}}(y)\right] \quad \forall y \in Y
$$

and by (17) we conclude that

$$
\tilde{B}=1 / \operatorname{FOU}(\tilde{B}) .
$$

The combined results of (61), (62), (57), and (58) are exactly the same as those in [9]; hence, we have been able to obtain the FOU of the T2 fired output FS using T1 FS mathematics.

\section{Singleton Fuzzification and Multiple Antecedents}

In the rule (46), let $\tilde{F}_{1}, \tilde{F}_{2}, \ldots, \tilde{F}_{p}$ be IT2 FSs in discrete universe of discourses $X_{1 d}, X_{2 d}, \ldots, X_{p d}$, respectively, and $\tilde{G}$ be an IT2 FS in universe of discourse $Y_{d}$. Decompose each $\tilde{F}_{i}$ into its $n_{F_{i}}(i=1, \ldots, p)$ embedded IT2 FSs $\tilde{F}_{i e}^{j_{i}}$, i.e.,

$$
\tilde{F}_{i}=\sum_{j_{i}=1}^{n_{F_{i}}} \tilde{F}_{i e}^{j_{i}}=1 / \operatorname{FOU}\left(\tilde{F}_{i}\right), \quad i=1, \ldots, p .
$$

The domain of each $\tilde{F}_{i e}^{j_{i}}$ is the embedded T1 FS $F_{i e}^{j_{i}}$. As in the preceding section, we decompose $\tilde{G}$ into $n_{G}$ embedded IT2 FSs $\tilde{G}_{e}^{j}$, whose domains are the embedded T1 FSs $G_{e}^{j}$, respectively, so (50) and (51) remain unchanged for this case.

The Cartesian product of $\tilde{F}_{1}, F_{2}, \cdots, \tilde{F}_{p}, \tilde{F}_{1} \times \tilde{F}_{2} \times \cdots \times \tilde{F}_{p}$, has $\prod_{i=1}^{p} n_{F_{i}}$ combinations of the embedded T1 FSs, $F_{i e}^{j_{i}}$. Let $F_{e}^{n}$ denote the $n$th combination of these embedded T1 FSs, i.e.,

$$
\begin{aligned}
F_{e}^{n} & =F_{1 e}^{j_{1}} \times \cdots \times F_{p e}^{j_{p}} \\
1 & \leq n \leq \prod_{i=1}^{p} n_{F_{i}} \text { and } 1 \leq j_{i} \leq n_{F_{i}} .
\end{aligned}
$$

This equation requires a combinatorial mapping from $\left(j_{1}, j_{2}, \ldots, j_{p}\right) \rightarrow n$; however, in the sequel we will not need to perform the specific mapping. All we need to understand is that it is theoretically possible to create such a mapping. To represent this mapping explicitly, we show $\left(j_{1}, j_{2}, \ldots, j_{p}\right) \rightarrow\left(j_{1}(n), j_{2}(n), \ldots, j_{p}(n)\right)$, so that (64) can be expressed as

$$
\begin{aligned}
F_{e}^{n} & =F_{1 e}^{j_{1}(n)} \times \cdots \times F_{p e}^{j_{p}(n)}, \\
& 1 \leq n \leq \prod_{i=1}^{p} n_{F_{i}} \text { and } 1 \leq j_{i}(n) \leq n_{F_{i}}
\end{aligned}
$$

in which case

$$
\begin{aligned}
\mu_{F_{e}^{n}}(\mathbf{x}) & =T_{m=1}^{p} \mu_{F_{m e}^{j_{m}(n)}}\left(x_{m}\right), \\
1 & \leq n \leq \prod_{i=1}^{p} n_{F_{i}} \text { and } 1 \leq j_{m}(n) \leq n_{F_{m}} .
\end{aligned}
$$

Additionally, let

$$
n_{F} \equiv \prod_{m=1}^{p} n_{F_{m}} .
$$

With $n_{G}$ embedded T1 FSs for the consequent, we obtain $n_{F} \times$ $n_{G}$ combinations of antecedent and consequent embedded T1
FSs, which generate the bundle of $n_{F} \times n_{G}$ fired output consequent T1 FS functions, i.e.,

$$
B(y)=\sum_{n=1}^{n_{F}} \sum_{j=1}^{n_{G}} \mu_{B(n, j)}(y) \quad \forall y \in Y_{d} .
$$

Observe how similar (68) and (52) are.

Theorem 3 is valid for this case, but in its proof the following changes must be made.

1) In (53), instead of computing $\mu_{B\left(j_{1}, j\right)}(y)$, we must now compute $\mu_{B(n, j)}(y)$, by using the top line of (44) in which $T_{m=1}^{p} \mu_{F_{m}^{l}}\left(x_{m}^{\prime}\right)$ is replaced by (66), i.e.,

$\mu_{B(n, j)}(y)=\left[T_{m=1}^{p} \mu_{F_{m e}^{j_{m}(n)}}\left(x_{m}^{\prime}\right)\right] \star \mu_{G^{j}}(y) \quad \forall y \in Y_{d}$.

2) Equation (54) is unchanged.

3) In (55) and (56), replace the index $j_{1}$ by the index $n$.

4) Let $\bar{\mu}_{\tilde{F}_{m}}\left(x_{m}\right)$ and $\underline{\mu}_{\tilde{F}_{m}}\left(x_{m}\right)$ denote the upper and lower MFs for $\tilde{F}_{m}$. Additionally, let $\bar{\mu}_{F_{m}}\left(x_{m}\right)$ and $\underline{\mu}_{F_{m}}\left(x_{m}\right)$ denote the embedded T1 FSs associated with $\bar{\mu}_{\tilde{F}_{m}}\left(x_{m}\right)$ and $\underline{\mu}_{\tilde{F}_{m}}\left(x_{m}\right)$, respectively. Note that $\bar{\mu}_{F_{m}}\left(x_{m}\right)$ and $\underline{\mu}_{F_{m}}\left(x_{m}\right)$ are two of the $n_{F_{m}}$ embedded T1 FSs that are associated with $\tilde{F}_{m}$. They will be the ones that are used in the next step.

5) Equations (57) and (58) are changed to

$$
\begin{aligned}
\underline{\mu}_{\tilde{B}}(y) & =\inf _{\forall n, j}\left(\mu_{B(n, j)}(y)\right) \\
& =\left[T_{m=1}^{p} \underline{\mu}_{F_{m}}\left(x_{m}^{\prime}\right)\right] \star \underline{\mu}_{G}(y) \quad \forall y \in Y_{d} \\
\bar{\mu}_{\tilde{B}}(y) & =\sup _{\forall n, j}\left(\mu_{B(n, j)}(y)\right) \\
& =\left[T_{m=1}^{p} \bar{\mu}_{F_{m}}\left(x_{m}^{\prime}\right)\right] \star \bar{\mu}_{G}(y) \quad \forall y \in Y_{d} .
\end{aligned}
$$

6) Equations (59)-(62) remain unchanged.

\section{Type-1 Nonsingleton Fuzzification and Multiple Antecedents}

The results for this case build upon the results for the just-considered case of singleton fuzzification and multiple antecedents. Equations (63)-(68) hold for the present case, and again (50) and (51) remain unchanged. Theorem 3 is also valid for this case, but in its proof the following changes must now be made.

1) For NSF, in order to compute $\mu_{B(n, j)}(y)$ in (68) we must use the second line of (44) in which ${ }^{13} \mu_{F_{m}}\left(x_{m}\right)$ is replaced by $\mu_{F_{m e}^{j_{m}(n)}}\left(x_{m}\right)$, i.e.,

$$
\begin{array}{r}
\mu_{B(n, j)}(y)=\left[T_{m=1}^{p}\left(\sup _{x_{m} \in X_{m}} \mu_{X_{m}}\left(x_{m}\right) \star \mu_{F_{m e}^{j_{m}(n)}}\left(x_{m}\right)\right)\right] \\
\star \mu_{G^{j}}(y) \quad \forall y \in Y_{d} .
\end{array}
$$

2)-4) Same as 2)-4) in Section V-C.

5) Equations (70) and (71) are changed to

$$
\begin{aligned}
\underline{\mu}_{\tilde{B}}(y) & =\inf _{\forall n, j}\left(\mu_{B(n, j)}(y)\right) \\
= & {\left[T_{m=1}^{p}\left(\sup _{x_{m} \in X_{m}} \mu_{X_{m}}\left(x_{m}\right) \star \underline{\mu}_{F_{m}}\left(x_{m}\right)\right)\right] } \\
& \star \underline{\mu}_{G}(y) \quad \forall y \in Y_{d}
\end{aligned}
$$

\footnotetext{
${ }^{13}$ Footnote 11 also applies here.
} 


$$
\begin{aligned}
\bar{\mu}_{\tilde{B}}(y)= & \sup _{\forall n, j}\left(\mu_{B(n, j)}(y)\right) \\
= & {\left[T_{m=1}^{p}\left(\sup _{x_{m} \in X_{m}} \mu_{X_{m}}\left(x_{m}\right) \star \bar{\mu}_{F_{m}}\left(x_{m}\right)\right)\right] } \\
& \star \bar{\mu}_{G}(y) \quad \forall y \in Y_{d} .
\end{aligned}
$$

6) Equations (59)-(62) again remain unchanged.

\section{E. Type-2 Nonsingleton Fuzzification and Multiple Antecedents}

The difference between this case and the previous one is the type-2 nonsingleton fuzzification, which, as we explain next, further increases the number of functions contained in the bundle of functions in (68). Most generally, the $p$-dimensional input to a rule is now given by the IT2 FS $\tilde{A}_{\mathbf{x}}$, where (as is commonly done) we assume a separable MF, and we let $\tilde{X}_{i}$ denote the labels of the IT2 FSs describing each of the $p$ inputs. More specifically, $\tilde{X}_{1}, \tilde{X}_{2}, \ldots, \tilde{X}_{p}$ are IT2 FSs in discrete universes of discourse $X_{1 d}, X_{2 d}, \ldots, X_{p d}$. Decompose $\tilde{X}_{i}$ into their $n_{X_{i}}(i=1, \ldots, p)$ embedded IT2 FSs $\tilde{X}_{i e}^{\gamma_{i}}$, i.e.,

$$
\tilde{X}_{i}=\sum_{\gamma_{i}=1}^{n_{X_{i}}} \tilde{X}_{i e}^{\gamma_{i}}, \quad i=1, \ldots, p .
$$

The domain of each $\tilde{X}_{i e}^{\gamma_{i}}$ is the embedded T1 FS $X_{i e}^{\gamma_{i}}$. The Cartesian product of $\tilde{X}_{1}, X_{2}, \ldots, \tilde{X}_{p}, \tilde{X}_{1} \times \tilde{X}_{2} \times \cdots \times \tilde{X}_{p}$, has $\prod_{\delta=1}^{p} n_{X_{\delta}}$ combinations of the embedded T1 FSs $X_{i e}^{\gamma_{i}}$. Let $X_{e}^{k}$ denote the $k$ th combination of these embedded T1 FSs, i.e.,

$$
\begin{aligned}
X_{e}^{k} & =X_{1 e}^{\gamma_{1}} \times \cdots \times X_{p e}^{\gamma_{p}} \\
1 & \leq k \leq \prod_{\delta=1}^{p} n_{X_{\delta}} \text { and } 1 \leq \gamma_{\delta} \leq n_{X_{\delta}} .
\end{aligned}
$$

This equation requires a combinatorial mapping from $\left(\gamma_{1}, \gamma_{2}, \ldots, \gamma_{p}\right) \rightarrow k$; however, in the sequel we will not need to perform the specific mapping. All we need to again understand is that it is theoretically possible to create such a combinatorial mapping. To represent this mapping explicitly, we show $\left(\gamma_{1}, \gamma_{2}, \ldots, \gamma_{p}\right) \rightarrow\left(\gamma_{1}(k), \gamma_{2}(k), \ldots, \gamma_{p}(k)\right)$, so that (76) can be expressed as

$$
\begin{aligned}
X_{e}^{k} & =X_{1 e}^{\gamma_{1}(k)} \times \cdots \times X_{p e}^{\gamma_{p}(k)} \\
1 & \leq k \leq \prod_{\delta=1}^{p} n_{X_{\delta}} \text { and } 1 \leq \gamma_{\delta} \leq n_{X_{\delta}}
\end{aligned}
$$

in which case

$$
\begin{aligned}
\mu_{X_{e}^{k}}(\mathbf{x}) & =T_{m=1}^{p} \mu_{X_{m e}}^{\gamma_{m}(k)}\left(x_{m}\right) \\
1 & \leq k \leq \prod_{\delta=1}^{p} n_{X_{\delta}} \text { and } 1 \leq \gamma_{m}(k) \leq n_{X_{m}} .
\end{aligned}
$$

Additionally, let

$$
n_{X} \equiv \prod_{\delta=1}^{p} n_{X_{\delta}} .
$$

There are still $n_{G}$ embedded T1 FSs for the consequent, $n_{F}=$ $\prod_{m=1}^{p} n_{F_{m}}$ embedded T1 FSs for the antecedents, and now $n_{X}$ embedded T1 FSs for the inputs; hence, we obtain $n_{X} \times n_{F} \times n_{G}$ combinations of input, antecedent and consequent embedded T1 FSs, which generate a bundle $B(y)$ of $n_{X} \times n_{F} \times n_{G}$ fired output consequent T1 FS functions, where now

$$
B(y)=\sum_{k=1}^{n_{X}} \sum_{n=1}^{n_{F}} \sum_{j=1}^{n_{G}} \mu_{B(k, n, j)}(y) .
$$

In order to again see the forest from the trees, we depict (80) in Fig. 10, for the simple case of a single-antecedent rule (in which case $n_{X}=n_{X_{1}}$ and $n_{F}=n_{F_{1}}$ ).

Theorem 3 is also valid for this case, but in its proof the following changes must now be made.

1) In order to compute $\mu_{B(n, j, k)}(y)$ in (80), we must again use the second line of (44) in which $\mu_{X_{m}}\left(x_{m}\right)$ and $\mu_{F_{m}}\left(x_{m}\right)$ are replaced by $\mu_{X_{m e}}^{\gamma_{m}(k)}\left(x_{m}\right)$ and $\mu_{F_{m e}}^{j_{m}(n)}\left(x_{m}\right)$, respectively, i.e.,

$$
\begin{aligned}
& \mu_{B(n, j, k)}(y) \\
& =\left[T_{m=1}^{p}\left(\sup _{x_{m} \in X_{m}} \mu_{X_{m e}^{\gamma m}(k)}\left(x_{m}\right) \star \mu_{F_{m e}^{j_{m}(n)}}\left(x_{m}\right)\right)\right] \\
& \quad \star \mu_{G^{j}}(y) \quad \forall y \in Y_{d} .
\end{aligned}
$$

2)-4a) Same as 2)-4) in Section V-C.

4b) Let $\bar{\mu}_{\tilde{X}_{m}}\left(x_{m}\right)$ and $\underline{\mu}_{\tilde{X}_{m}}\left(x_{m}\right)$ denote the upper and lower MFs for $\tilde{X}_{m}$. Additionally, let $\bar{\mu}_{X_{m}}\left(x_{m}\right)$ and $\underline{\mu}_{X_{m}}\left(x_{m}\right)$ denote the embedded T1 FSs associated with $\bar{\mu}_{\tilde{X}_{m}}\left(x_{m}\right)$ and $\underline{\mu}_{\tilde{X}_{m}}\left(x_{m}\right)$, respectively. Note that $\bar{\mu}_{X_{m}}\left(x_{m}\right)$ and $\underline{\mu}_{X_{m}}\left(x_{m}\right)$ are two of the $n_{X_{m}}$ embedded T1 FSs that are associated with $\tilde{X}_{m}$. They will be the ones used in the next step.

5) Equations (73) and (74) are changed to

$$
\begin{aligned}
\underline{\mu}_{\tilde{B}}(y)= & \inf _{\forall k, n, j}\left(\mu_{B(k, n, j)}(y)\right) \\
= & {\left[T_{m=1}^{p}\left(\sup _{x_{m} \in X_{m}} \underline{\mu}_{X_{m}}\left(x_{m}\right) \star \underline{\mu}_{F_{m}}\left(x_{m}\right)\right)\right] } \\
& \star \underline{\mu}_{G}(y) \quad \forall y \in Y \\
\bar{\mu}_{\tilde{B}}(y)= & \sup _{\forall k, n, j}\left(\mu_{B(k, n, j)}(y)\right) \\
= & {\left[T_{m=1}^{p}\left(\sup _{x_{m} \in X_{m}} \bar{\mu}_{X_{m}}\left(x_{m}\right) \star \bar{\mu}_{F_{m}}\left(x_{m}\right)\right)\right] } \\
& \star \bar{\mu}_{G}(y) \quad \forall y \in Y .
\end{aligned}
$$

6) Again, (59)-(62) remain unchanged.

\section{F. Multiple Rules}

So far, all of the derivations in Sections V-B-G have been for a single rule. In general, there are $M$ rules that characterize an IT2 FLS, and frequently more than one rule fires when input $\mathbf{x}$ is applied to that system. What this means is that, as in the case of a T1 FLS, we need to include another index $-l$ - in all of the IT2 FLS formulas. So, for example, for the $l$ th rule, we would express (62), (61), (82), and (83) as $(l=1, \ldots, M)$

$$
\begin{aligned}
\tilde{B}^{l} & =1 / \operatorname{FOU}\left(\tilde{B}^{l}\right) \\
\operatorname{FOU}\left(\tilde{B}^{l}\right) & =\left[\underline{\mu}_{\tilde{B}^{l}}(y), \bar{\mu}_{\tilde{B}^{l}}(y)\right] \quad \forall y \in Y
\end{aligned}
$$




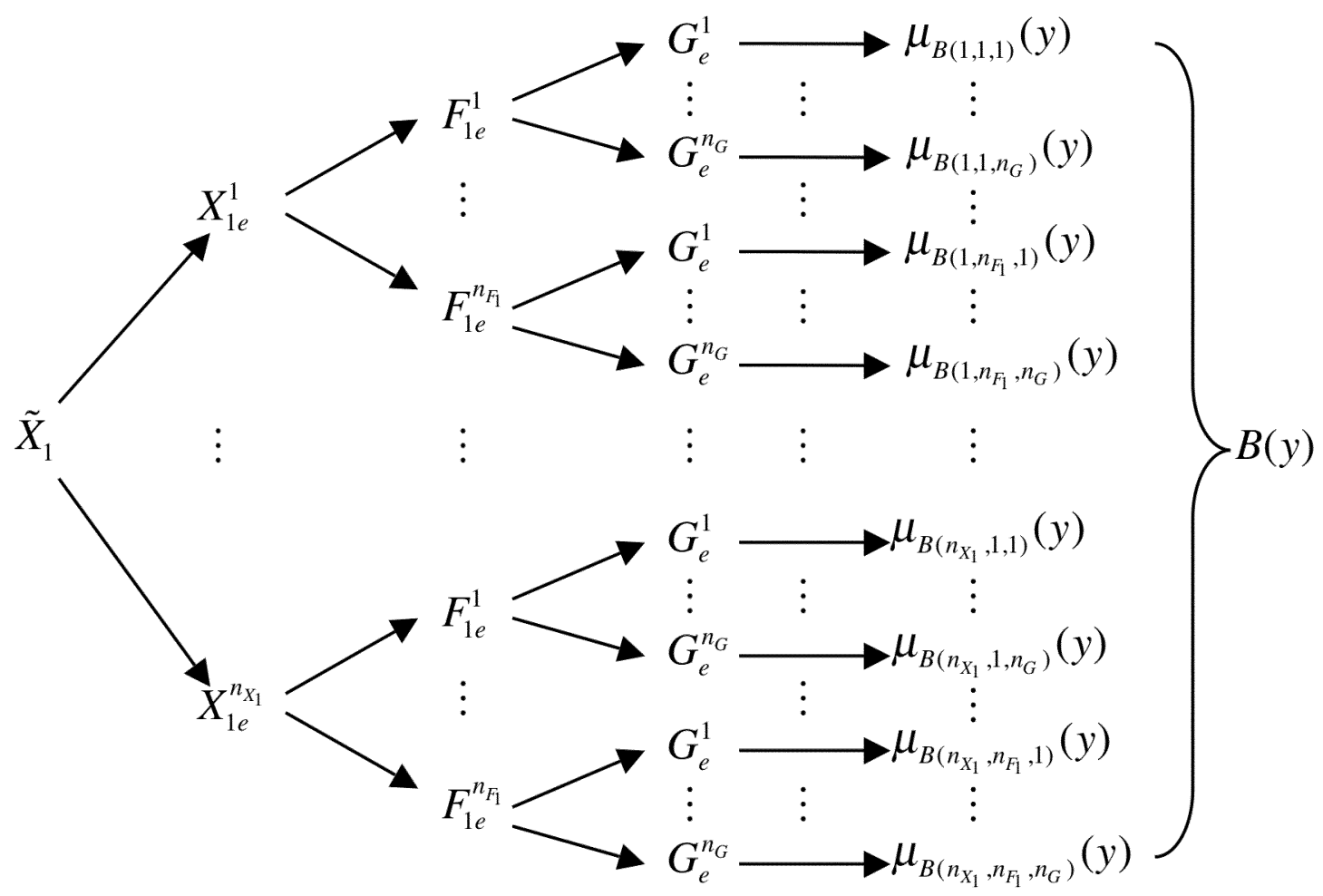

Fig. 10. Fired output FSs for all possible $n_{B}=n_{X_{1}} \times n_{F_{1}} \times n_{G}$ combinations of the embedded T1 antecedent and consequent FSs for a single antecedent rule.

$$
\begin{aligned}
\underline{\mu}_{\tilde{B}^{l}}(y)= & \inf _{\forall k, n, j}\left(\mu_{B^{l}(k, n, j)}(y)\right) \\
= & {\left[T_{m=1}^{p}\left(\sup _{x_{m} \in X_{m}} \underline{\mu}_{X_{m}}\left(x_{m}\right) \star \underline{\mu}_{F_{m}^{l}}\left(x_{m}\right)\right)\right] } \\
& \star \underline{\mu}_{G^{l}}(y) \quad \forall y \in Y \\
\bar{\mu}_{\tilde{B}^{l}}(y)= & \sup _{\forall k, n, j}\left(\mu_{B^{l}(k, n, j)}(y)\right) \\
= & {\left[T_{m=1}^{p}\left(\sup _{x_{m} \in X_{m}} \bar{\mu}_{X_{m}}\left(x_{m}\right) \star \bar{\mu}_{F_{m}^{l}}\left(x_{m}\right)\right)\right] } \\
& \star \bar{\mu}_{G^{l}}(y) \quad \forall y \in Y .
\end{aligned}
$$

As in the $\mathrm{T} 1$ case, fired rule sets are combined either before or as part of output processing. For illustrative purposes only, ${ }^{14}$ let us assume that the $l$ fired rule sets are combined using the union operation. In this case, we have the following.

Theorem 4: If the $l$ fired rule sets are combined using the union operation, leading to a composite IT2 FS $\tilde{B}$, then

$$
\begin{aligned}
\tilde{B} & =1 / \operatorname{FOU}(\tilde{B}) \\
\operatorname{FOU}(\tilde{B}) & =\left[\underline{\mu}_{\tilde{B}}(y), \bar{\mu}_{\tilde{B}}(y)\right] \quad \forall y \in Y
\end{aligned}
$$

where

$$
\begin{aligned}
& \underline{\mu}_{\tilde{B}}(y)=\underline{\mu}_{\tilde{B}^{1}}(y) \vee \underline{\mu}_{\tilde{B}^{2}}(y) \vee \cdots \vee \underline{\mu}_{\tilde{B}^{M}}(y) \\
& \bar{\mu}_{\tilde{B}}(y)=\bar{\mu}_{\tilde{B}^{1}}(y) \vee \bar{\mu}_{\tilde{B}^{2}}(y) \vee \cdots \vee \bar{\mu}_{\tilde{B}^{M}}(y)
\end{aligned}
$$

and specific formulas for $\underline{\mu}_{\tilde{B}^{l}}(y)$ and $\bar{\mu}_{\tilde{B}^{l}}(y)$ are given in Sections V-B-F.

\footnotetext{
${ }^{14}$ We do not necessarily advocate combining IT2 FSs using the union operation, just as many people do not advocate combining fired T1 FSs in a T1 FLS using the union operation. This is explained in great detail in [17] where more computationally tractable ways of blending the IT2 fired rule sets are described. Conceptually, one merely needs to think of some final (aggregated) IT2 FS, say $\tilde{B}(y)$ as having been obtained from the $\tilde{B}^{l}(y)$.
}

Proof: Equations (90) and (91) follow from $M-1$ repeated applications of (21) to $\bigcup_{l=1}^{M} \tilde{B}^{l}$.

\section{G. Output Processing}

With reference to the T2 FLS depicted in Fig. 8, we now explain how to perform output processing. Type-reduction, the first step of output processing computes the centroid of an IT2 FS, where the specific IT2 FS that it does this for is one that is associated with the IT2 fired-rule output FSs whose formulas have just been obtained in Sections V-B-F. We are motivated to compute the centroid of an IT2 FS because when all sources of uncertainty that are present in an IT2 FLS disappear, we require that the IT2 FLS must reduce to a T1 FLS, and many T1 defuzzification methods are based on computing the centroid of a T1 FS. Because computing the centroid of an IT2 FS is so important, we provide a brief description of it next.

Using Representation Theorem 1, we define the centroid, $C_{\tilde{B}}$, of an IT2 FS $\tilde{B}$ as the collection of the centroids of all of its embedded IT2 FSs. From (17), and the first part of (18), we see that this means we need to compute the centroids of all of the $n_{B}$ embedded T1 FSs contained within $\mathrm{FOU}(\tilde{B})$. The results of doing this will be a collection of $n_{B}$ numbers, and these numbers will have both a smallest and largest element, $c_{l}(\tilde{B}) \equiv c_{l}$ and $c_{r}(\tilde{B}) \equiv c_{r}$, respectively. That such numbers exist is because the centroid of each of the embedded T1 FSs is a bounded number. Associated with each of these numbers will be a membership grade of 1, because the secondary grades of an IT2 FS are all equal to 1 . This means

$$
C_{\tilde{B}}=1 /\left\{c_{l}, \ldots, c_{r}\right\}
$$


where ${ }^{15}$

$$
\begin{gathered}
c_{l}=\min _{\forall \theta_{i} \in\left[\underline{\mu}_{\tilde{B}}\left(y_{i}\right), \bar{\mu}_{\tilde{B}}\left(y_{i}\right)\right]} \frac{\sum_{i=1}^{N} y_{i} \theta_{i}}{\sum_{i=1}^{N} \theta_{i}} \\
c_{r}=\max _{\forall \theta_{i} \in\left[\underline{\mu}_{\tilde{B}}\left(y_{i}\right), \bar{\mu}_{\tilde{B}}\left(y_{i}\right)\right]} \frac{\sum_{i=1}^{N} y_{i} \theta_{i}}{\sum_{i=1}^{N} \theta_{i}} .
\end{gathered}
$$

In general, there are no closed-form formulas for $c_{l}$ and $c_{r}$; however, Karnik and Mendel [7] have developed two very simple and easy to implement iterative algorithms for computing these end-points exactly, and they can be run in parallel. Although space does not permit us to provide the details of these algorithms here, we state the resulting general formulas for $c_{l}$ and $c_{r}$

$$
\begin{gathered}
c_{l}=\frac{\sum_{i=1}^{L} y_{i} \bar{\mu}_{\tilde{B}}\left(y_{i}\right)+\sum_{i=L+1}^{N} y_{i} \underline{\mu}_{\tilde{B}}\left(y_{i}\right)}{\sum_{i=1}^{L} \bar{\mu}_{\tilde{B}}\left(y_{i}\right)+\sum_{i=L+1}^{N} \underline{\mu}_{\tilde{B}}\left(y_{i}\right)} \\
c_{r}=\frac{\sum_{i=1}^{R} y_{i} \underline{\mu}_{\tilde{B}}\left(y_{i}\right)+\sum_{i=R+1}^{N} y_{i} \bar{\mu}_{\tilde{B}}\left(y_{i}\right)}{\sum_{i=1}^{R} \underline{\mu}_{\tilde{B}}\left(y_{i}\right)+\sum_{i=R+1}^{N} \bar{\mu}_{\tilde{B}}\left(y_{i}\right)} .
\end{gathered}
$$

In these formulas, $L$ and $R$ are switch points and it is these two points that are determined iteratively by the Karnik-Mendel algorithms.

Note that we have stated (92)-(96) in the context of centroid type-reduction; however, there are as many type-reduction methods as there are T1 defuzzification methods, because each of the former is associated with one of the latter. Karnik and Mendel [7] have also developed center-of-sums, height, modified-height and center-of-sets type-reducers, for which the symbols in (92)-(96) take on different meanings. See [17] for a careful explanation of these different kinds of type-reduction.

Regardless of which type-reduction method one uses, defuzzification-which follows type-reduction-is based on using the average of $c_{l}$ and $c_{r}$, i.e.,

$$
y(\mathbf{x})=\frac{1}{2}\left[c_{l}(\mathbf{x})+c_{r}(\mathbf{x})\right] .
$$

We have now completed all of the computations that characterize an IT2 FLS.

\section{H. Comments}

It is worth reiterating that although we used the concept of an embedded T1 FS to derive the fired-rule output FS of various kinds of IT2 FLSs, we never actually had to compute the $n_{X} \times$ $n_{F} \times n_{G} \mu_{B(k, n, j)}(y)$, a number of computations that could be astronomical. Instead, in all cases, we showed that we only need to compute two functions, $\underline{\mu}_{\tilde{B}^{l}}(y)$ and $\bar{\mu}_{\tilde{B}^{l}}(y)$. It is this

\footnotetext{
${ }^{15}$ When discretizations of the primary variable and primary membership approach zero, $\left\{c_{l}, \ldots, c_{r}\right\} \rightarrow\left[c_{l}, c_{r}\right]$, an interval set. In the literature about the centroid (e.g., [7] and [17]), it is customary to see (92) written as $C_{\tilde{B}}=\left[c_{l}, c_{r}\right]$.
}

tremendous reduction in computations that distinguishes an IT2 FLS from a general T2 FLS. For the latter, one must not only compute the FOU of each fired rule but also the secondary grade at each value of $y$. At present, such calculations are not practical, but in the future, research on efficient ways to perform them may make them practical (e.g., [1]). Such research must not only address the calculations of the fired-rule T2 FSs but also typereduction for general T2 FSs.

After all is said and done, we observe from the various formulas that we have derived for $\underline{\mu}_{\tilde{B}^{l}}(y)$ and $\bar{\mu}_{\tilde{B}^{l}}(y)$ [e.g., (86) and (87)] that they each only make use of the respective lower or upper MFs of input, antecedent and consequent IT2 FSs. This suggests that an IT2 FLS is performing a worst-case design - worst case in the sense that $\underline{\mu}_{\tilde{B}^{l}}(y)$ uses just the lower MFs of input, antecedent and consequent IT2 FSs, whereas $\bar{\mu}_{\tilde{B}^{l}}(y)$ uses just the upper MFs of such IT2 FSs. Most importantly, these two calculations are totally uncoupled, i.e., there is no sharing of lower or upper MF values across them.

If the final output of the IT2 FLS is just some combination of its fired output IT2 FSs [as in (88)-(91)], then that output would indeed represent a worst-case design. If, however, the final output is a number, computed as just described in Section V-G, then by examining (95) and (96) we see that it is through the process of type-reduction (i.e., a centroid calculation) that upper and lower MF information is shared, making the resulting IT2 FLS more than just a worst-case design. Sharing can also be accomplished without the Karnik-Mendel kind of type-reduction by using an ad hoc type-reduction formula for output $y(\mathbf{x})$ (such as in [3]), e.g., 16

$$
y(\mathbf{x}) \equiv \frac{1}{2}\left[\underline{\mu}_{\tilde{B}}(y)+\bar{\mu}_{\tilde{B}}(y)\right] .
$$

Which way of sharing upper and lower MF information (there can also be other ways) is better is an open question.

Finally, in (44) the bracketed terms that involve $p-1 \mathrm{t}$-norms is often referred to as a firing level, $f(\mathbf{x})$. For an IT2 FLS the firing level becomes a firing interval, e.g., for SF, examining (61), (70) and (71), it is easy to see that if

$$
F\left(\mathbf{x}^{\prime}\right) \equiv\left[\underline{f}\left(\mathbf{x}^{\prime}\right), \bar{f}\left(\mathbf{x}^{\prime}\right)\right]
$$

denotes a firing interval, where

$$
\begin{aligned}
\underline{f}\left(\mathbf{x}^{\prime}\right) & \equiv T_{m=1}^{p} \underline{\mu}_{F_{m}}\left(x_{m}^{\prime}\right) \\
\bar{f}\left(\mathbf{x}^{\prime}\right) & \equiv T_{m=1}^{p} \bar{\mu}_{F_{m}}\left(x_{m}^{\prime}\right)
\end{aligned}
$$

then

$$
\begin{aligned}
\operatorname{FOU}(\tilde{B}) & =\left[\underline{\mu}_{\tilde{B}}(y), \bar{\mu}_{\tilde{B}}(y)\right] \\
& =\left[\underline{f}\left(\mathbf{x}^{\prime}\right) \star \mu_{G}(y), \bar{f}\left(\mathbf{x}^{\prime}\right) \star \bar{\mu}_{G}(y)\right] \\
& =\left[\underline{f}\left(\mathbf{x}^{\prime}\right), \bar{f}\left(\mathbf{x}^{\prime}\right)\right] \star\left[\underline{\mu}_{G}(y), \bar{\mu}_{G}(y)\right] \quad \forall y \in Y_{d} .
\end{aligned}
$$

We leave it to the reader to show that (102) also applies to all of the cases we have considered in Sections V-B-F, modulo appropriate modifications for $f\left(\mathbf{x}^{\prime}\right)$ and $\bar{f}\left(\mathbf{x}^{\prime}\right)$.

\footnotetext{
${ }^{16}$ Note that, although not explicitly shown $\underline{\mu}_{\tilde{B}}(y)$, and $\bar{\mu}_{\tilde{B}}(y)$ depend upon $\mathbf{x}$, e.g., see (86) and (87), which is why we have used $y(\mathbf{x})$ in (97) and (98).
} 
It is often useful to use the notion of a firing interval when contrasting an IT2 FLS with a T1 FLS. Note that when all sources of uncertainty disappear $f\left(\mathbf{x}^{\prime}\right) \rightarrow \bar{f}\left(\mathbf{x}^{\prime}\right)=f\left(\mathbf{x}^{\prime}\right)$ and $\underline{\mu}_{G}(y) \rightarrow \bar{\mu}_{G}(y)=\mu_{G}(y)$, in which case the IT2 FLS results in (100)-(102) reduce to the T1 FLS results in (44).

\section{CONCLUSION}

We have shown that all of the results that are needed to implement an IT2 FLS can be obtained using T1 FS mathematics. The key to doing this is the Mendel-John Representation Theorem for a T2 FS. We can now develop an IT2 FLS in a much more straightforward way. Since an IT2 FLS models higher levels of uncertainty than does a T1 FLS, this opens up an efficient way of developing improved control systems and for modeling human decision making.

We believe that the results in this paper will make IT2 FLSs much more accessible to practitioners of FL since the time and effort now required to learn about IT2 FLSs is very small. We also believe that the approach taken in this paper can be used to extend many existing T1 FS results to IT2 FSs. Whether or not comparable results can be obtained for general T2 FLSs is an open question.

\section{REFERENCES}

[1] S. Coupland and R. John, "A new and efficient method for the type-2 meet operation," in Proc. FUZZ-IEEE, Budapest, Hungary, Jul. 2004, pp. 959-964.

[2] M. Gehrke, C. Walker, and E. Walker, "Some comments on interval valued fuzzy sets,” Int. J. Intell. Syst., vol. 11, pp. 751-759, 1996.

[3] M. B. Gorzalczany, "Interval-valued fuzzy controller based on verbal model of object," Fuzzy Sets Syst., vol. 28, pp. 45-53, 1988.

[4] H. Hagras, "A hierarchical type-2 fuzzy logic control architecture for autonomous mobile robots," IEEE Trans. Fuzzy Syst., vol. 12, no. 4, pp. 524-539, Aug. 2004.

[5] J.-S. R. Jang and C.-T. Sun, "Neuro-fuzzy modeling and control," Proc. IEEE, vol. 83, no. 3, pp. 378-406, Mar. 1995.

[6] J.-S. R. Jang, C.-T. Sun, and E. Mizutanni, Neuro-Fuzzy and Soft-Computing. Upper Saddle River, NJ: Prentice-Hall, 1997.

[7] N. N. Karnik and J. M. Mendel, "Centroid of a type-2 fuzzy set," Inform. Sci., vol. 132, pp. 195-220, 2001.

[8] G. J. Klir and B. Yuan, Fuzzy Sets and Fuzzy Logic: Theory and Applications. Upper Saddle River, NJ: Prentice-Hall, 1995.

[9] Q. Liang and J. M. Mendel, "Interval type-2 fuzzy logic systems: Theory and design," IEEE Trans. Fuzzy Syst., vol. 8, no. 5, pp. 535-550, Oct. 2000.

[10] — - "Equalization of nonlinear time-varying channels using type-2 fuzzy adaptive filters," IEEE Trans. Fuzzy Syst., vol. 8, no. 5, pp. 551-563, Oct. 2000.

[11] - "Overcoming time-varying co-channel interference using type-2 fuzzy adaptive filter," IEEE Trans. Circuits Syst., vol. 9, no. 6, pp. 1419-1428, Dec. 2000.

[12] - "MPEG VBR video traffic modeling and classification using fuzzy techniques," IEEE Trans. Fuzzy Syst., vol. 9, no. 1, pp. 183-193, Feb. 2001

[13] Q. Liang, N. N. Karnik, and J. M. Mendel, "Connection admission control in ATM networks using survey-based type-2 fuzzy logic systems," IEEE Trans. Syst., Man, Cybern., C, Appl. Rev., vol. 30, no. 3, pp. 329-339, Aug. 2000.

[14] M. C. Melgarejo, A. P.-Reyes, and A. Garcia, "Computational model and architectural proposal for a hardware type-2 fuzzy system," in Proc. IEEE FUZZ Conf., Budapest, Hungary, Jul. 2004.

[15] P. Melin and O. Castillo, "A new method for adaptive control of nonlinear plants using type-2 fuzzy logic and neural networks," Int. J. Gen. Syst., vol. 33, pp. 289-304, 2004.

[16] J. M. Mendel, "On the importance of interval sets in type-2 fuzzy logic systems," in Proc. Joint 9th IFSA World Congr. 20th NAFIPS Int. Conf., Vancouver, BC, Canada, Jul. 25-28, 2001, pp. 1647-1652.

[17] —, Uncertain Rule-Based Fuzzy Logic Systems: Introduction and New Directions. $\quad$ Upper Saddle River, NJ: Prentice-Hall, 2001.
[18] — "Type-2 fuzzy sets: Some questions and answers," IEEE Connections, vol. 1, pp. 10-13, Aug. 2003

[19] J. M. Mendel and R. I. B. John, "Type-2 fuzzy sets made simple," IEEE Trans. Fuzzy Syst., vol. 10, no. 2, pp. 117-127, Apr. 2002.

[20] - "Footprint of uncertainty and its importance to type-2 fuzzy sets," in Proc. 6th IASTED Int. Conf. Artificial Intelligence and Soft Computing, Banff, Canada, Jul. 2002, pp. 587-592.

[21] T. Ozen and J. M. Garibaldi, "Investigating adaptation in type-2 fuzzy logic systems applied to umbilical acid-base assessment," in Proc. Eur. Symp. Intelligent Technologies, Oulu, Finland, Jul. 2003, pp. 289-294.

[22] D. Wu and W. Tan, "A type-2 fuzzy logic controller for the liquid-level process," in Proc. IEEE Int. Conf. Fuzzy Systems, Budapest, Hungary, Jul. 2004

[23] H. Wu and J. M. Mendel, , E. M. Carapressa, Ed., "Classifier designs for binary classifications of ground vehicles," in Unattended Ground Sensor Technologies and Applications V. Orlando, FL: SPIE, 2003, vol. 5090, Proc. SPIE, pp. 122-133.

[24] L. A. Zadeh, "The concept of a linguistic variable and its application to approximate reasoning-1," Inform. Sci., vol. 8, pp. 199-249, 1975.

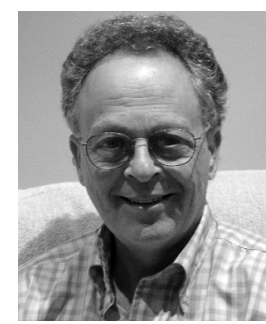

Jerry M. Mendel (S'59-M'61-SM'72-F'78LF'04) received the Ph.D. in electrical engineering from the Polytechnic Institute of Brooklyn, NY.

Currently he is Professor of Electrical Engineering at the University of Southern California, Los Angeles, where he has been since 1974. He has published over 470 technical papers and is author and/or editor of eight books, including Uncertain Rule-based Fuzzy Logic Systems: Introduction and New Directions (Prentice-Hall, 2001). His present research interests include: type-2 fuzzy logic systems and their applications to a wide range of problems, including target classification, smart oil field technology, and computing with words.

Dr. Mendel is a Distinguished Member of the IEEE Control Systems Society. He was President of the IEEE Control Systems Society in 1986, and is presently Chairman of the Fuzzy Systems Technical Committee and a member of the Administrative Committee of the IEEE Computational Intelligence Society. Among his awards are the 1983 Best Transactions Paper Award of the IEEE Geoscience and Remote Sensing Society, the 1992 Signal Processing Society Paper Award, the 2002 TRANSACTIONS ON FUZZY SYSTEMS Outstanding Paper Award, a 1984 IEEE Centennial Medal, and an IEEE Third Millenium Medal.

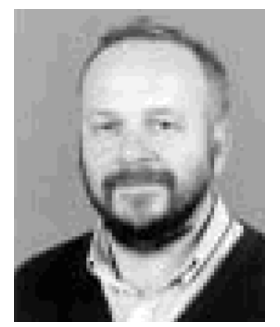

Robert I. John (M'01) received the B.Sc. (Hons.) degree in mathematics from Leicester Polytechnic, Leicester, U.K., the M.Sc. degree in statistics from UMIST, Manchester, U.K., and the Ph.D. degree in type-2 fuzzy logic De Montfort University, Leicester, U.K., in 1979, 1981, and 2000, respectively.

$\mathrm{He}$ is currently Director of the Centre for Computational Intelligence (CCI) at De Montfort University. The CCI specializes in using soft computing techniques for tackling difficult problems, as well as developing theoretical ideas in fuzzy logic. He has published over 100 papers on fuzzy logic and has edited three books. His research interests relate to the role of type- 2 fuzzy logic in modeling perceptions and, in particular, for modeling medical expertise.

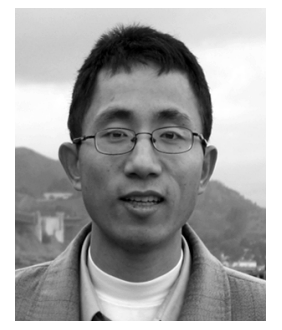

Feilong Liu (S'02) received his B.S. and M.S in automation theory and engineering from Chinese Northeastern University, Shenyang, Liaoning Province, P. R. China, and South China University of Technology, Guangzhou, Guangdong Province, P. R. China, in 1995 and 2000, respectively. He is currently working toward the Ph.D. degree in Electrical Engineering at the University of Southern California, Los Angeles.

His current research interests include type-2 fuzzy logic theory, artificial intelligence, signal processing, pattern recognition, and applying these technologies to smart oil field problems, such as water flooding. 